\title{
THEOREMS OF HARDY AND PALEY FOR VECTOR-VALUED ANALYTIC FUNCTIONS AND RELATED CLASSES OF BANACH SPACES
}

\author{
O. BLASCO AND A. PELCZYNSKI
}

\begin{abstract}
We investigate the classes of Banach spaces where analogues of the classical Hardy inequality and the Paley gap theorem hold for vector-valued functions. We show that the vector-valued Paley theorem is valid for a large class of Banach spaces (necessarily of cotype 2) which includes all Banach lattices of cotype 2, all Banach spaces whose dual is of type 2 and also the preduals of $C^{*}$-algebras. For the trace class $S_{1}$ and the dual of the algebra of all bounded operators on a Hilbert space a stronger result holds; namely, the vector-valued analogue of the Fefferman theorem on multipliers from $H^{1}$ into $l^{1}$; in particular for the latter spaces the vector-valued Hardy inequality holds. This inequality is also true for every Banach space of type $>1$ (Bourgain).
\end{abstract}

\section{INTRODUCTION}

If $f=\sum_{j \geq 0} a_{j} e^{i j t}$ is an analytic trigonometric polynomial, then

$$
\begin{aligned}
& \sum_{j \geq 0}\left|a_{j}\right|(j+1)^{-1} \leq C_{1} \int_{-\pi}^{\pi}|f(t)| d t, \\
& \left(\sum_{k \geq 0}\left|a_{2^{k}}\right|^{2}\right)^{1 / 2} \leq C_{2} \int_{-\pi}^{\pi}|f(t)| d t,
\end{aligned}
$$

where $C_{1}$ and $C_{2}$ are numerical constants independent of $f$ (cf. [Du]). The first fact is called the Hardy inequality; the second is a particular case of a theorem of Paley where $\left(2^{k}\right)$ is replaced by any sequence $\left(n_{k}\right)$ of positive integers with $\inf _{k} n_{k+1} / n_{k}>1$. Both inequalities are false if analytic trigonometric polynomials are replaced by arbitrary trigonometric polynomials; their proofs

Received by the editors October 15, 1987 and, in revised form, January 20, 1989.

1980 Mathematics Subject Classification (1985 Revision). Primary 46B20, 46E40; Secondary 46E15, 42A45, 46L05.

Key words and phrases. Hardy inequality, Paley theorem, multipliers, $X$-atoms, Banach spaces of type $>1$, cotype 2 .

The first author was supported in part by grant C.A.I.C.Y.T. PB85-0338; the second author was supported by SERC grant GR/DG68872 while visiting the University of Cambridge during the fall of 1986; both authors were supported by the University of Illinois at Urbana-Champaign while participating in the Special Year in Modern Analysis 1986/87. 
depend on special properties of the Hardy space $H^{1}$ like the factorization theorem and atomic decomposition.

In the present paper we are interested in finding under what additional conditions on a Banach space $X$ the inequalities remain true if the Fourier coefficients $a_{j}$ 's are elements of $X$ and absolute values are everywhere replaced by norms. In that setting for arbitrary Banach spaces the inequalities are false. A standard counterexample is the space $c_{0}$; a more sophisticated one $L^{1} / \bar{H}_{0}^{1}$ (see Proposition 1.1 for details). It appears that the validity of $X$-valued Hardy and Paley inequalities depends on geometric properties of $X$ like the (Rademacher) type and cotype or the Fourier type introduced by Peetre [Pee] (i.e. a vectorvalued version of the Hausdorff-Young inequality). Sometimes it depends on special analytic structure of $X$ like being a dual of a $C^{*}$-algebra. Obviously the validity of any of these inequalities or related ones is an invariant of the socalled "local theory of Banach spaces"; in particular, the validity for $X$ implies the validity for every Banach space finitely representable in $X$.

The paper consists of four sections. The first has a preliminary character. We recall the definitions of vector-valued Hardy spaces $H_{X}^{p}$ and the vector-valued atomic space $H_{X}^{1}$, at . The important discovery of Bourgain and Garcia-Cuerva (cf. Theorem 1.1) links $H_{X}^{1}$, at with $H_{X}^{1}$. Next we set up a framework for the vector-valued analogues of the Hardy and Paley inequalities; we rephrase them in terms of boundedness of operators induced by some scalar multipliers (for instance the sequence $\left((j+1)^{-1}\right)$ corresponds to the Hardy inequality). We also recall some basic facts from the local theory of Banach spaces.

$\S 2$ is devoted to the study of operators induced by bounded multipliers from $H^{1}$ into $l^{1}$ (the symbols $H^{1}$ and $l^{1}$ without subscripts denote the usual scalarvalued spaces). Obviously every scalar sequence which is a bounded multiplier from $H_{X}^{1}$ into $l_{X}^{1}$ for some Banach space $X$ is automatically a bounded multiplier from $H^{1}$ into $l^{1}$. We introduce the class of Banach spaces $X$ (called spaces of $\left(H^{1}-l^{1}\right)$-Fourier type, such that every bounded multiplier from $H^{1}$ into $l^{1}$ induces a bounded multipliers from $H_{X}^{1}$ into $l_{X}^{1}$. This class is a proper subclass of the Banach spaces of Cotype 2. We show that if $1 \leq p \leq 2$ then $L^{p}(\mu)$ and the Schatten-von Neumann trace class $S_{p}$ are spaces of $\left(H_{X}^{1}-l_{X}^{1}\right)$ Fourier type (cf. Corollary 2.2 and Theorem 2.2). The case of $S_{p}$ heavily depends on a noncommutative factorization theorem (cf. [S]). The case of $S_{1}$ yields that the dual space of the space of all linear operators on a Hilbert space is a space of $\left(H^{1}-l^{1}\right)$-Fourier type. Next we introduce the one-parameter family $F M_{q}$ for $1 \leq q \leq 2$ of sequence spaces which "connect" the class $F M_{1}=l^{1}$ of trivial multipliers with the class of all bounded multipliers from $H^{1}$ into $l^{1}$. The Hardy multiplier $\left((j+1)^{-1}\right)$ belongs to the intersection $\bigcap_{q>1} F M_{q}$. We show that if for a Banach space $X$ the vector-valued Hausdorff-Young inequality holds with an exponent $p \in[1,2]$ then every multiplier from $F M_{p}$ induces a bounded operator from $H_{X}^{1}$, at into $l_{X}^{1}$ (Theorem 2.4). As an appli- 
cation of this result we get that if $p>2$ and $E_{p}$ denotes either $L^{p}$ or $S_{p}$ then the class of scalar sequences inducing bounded operators from $H_{E_{p}}^{1}$ into $l_{E_{p}}^{1}$ coincides with $F M_{p}$ (Corollary 2.6). We also present a proof of a result due to J. Bourgain (Theorem 2.5) that if $X$ has type $>1$ then the $X$-valued Hardy inequality holds.

In $\S 3$ we study "Paley spaces", i.e. Banach spaces for which the vector-valued Paley theorem holds. This property is equivalent to the formally stronger one that every bounded multiplier from $H^{1}$ into $l^{1}$ induces a bounded operator for corresponding vector-valued spaces. The class of Paley spaces contains the class of $\left(H^{1}-l^{1}\right)$-Fourier type spaces and is contained as a proper subclass in the class of Banach spaces of cotype 2. However, for Banach lattices (Theorem 3.2) as well as for Banach spaces of type $>1$ (Proposition 3.5) the class of Paley spaces coincides with the class of Banach spaces of cotype 2 . Actually the vector-valued Paley theorem for $H_{X}^{1, \text { at }}$ characterizes Banach spaces of cotype 2 and type $>1$ (Proposition 3.5). Finally we show that the dual of a $C^{*}$-algebra is a Paley space (Theorem 3.3).

$\S 4$ contains a few open problems and some additional results. In particular we discuss some characterizations of Banach spaces of type $>1$ and the relationship between Paley spaces and 2-uniformly PL convexifiable spaces.

Acknowledgment. We would like to express our gratitude to D. J. H. Garling for valuable discussions in the early stage of this research concerning the cases of Banach lattices and $C^{*}$-algebras; we are particularly indebted to J. Bourgain for this permission to include Theorem 2.5 and to G. Pisier for several comments and remarks which improved the preliminary version of the paper. We would like also to thank the University of Illinois at Urbana Champaign, the University of Cambridge and St. John's College Cambridge for their hospitality and support.

\section{Preliminaries}

1.1 Vector-valued Hardy spaces $H_{X}^{p}$. Unless otherwise indicated all Banach spaces are considered to be taken over the complex number field $\mathbb{C}$. Given a Banach space $X$ and $p \in[1: \infty$ ) (respectively $p=\infty$ ) we denote by $L_{X}^{p}$ the space of all $X$-valued $2 \pi$-periodic functions on the real line $\mathbb{R}$ which are Bochner absolutely integrable in the $p$ th power (respectively essentially bounded) under the norm

$$
\|f\|_{p}=\left[(2 \pi)^{-1} \int_{-\pi}^{\pi}\|f(t)\|^{p} d t\right]^{1 / p} \text { for } 1 \leq p<\infty
$$

(respectively $\|f\|_{\infty}=\operatorname{ess~sup}_{t \in \mathbb{R}}\|f(t)\|$ ). by

Given $f \in L_{X}^{1}$ and an integer $j$, the $j$ th Fourier coefficient of $f$ is defined

$$
\hat{f}(j)=(2 \pi)^{-1} \int_{-\pi}^{\pi} e^{-i j t} f(t) d t .
$$


If for some nonnegative integer $n, \hat{f}(j)=0$ for $|j|>n$, then $f$ is called an $X$-valued trigonometric polynomial of degree $\leq n$; if moreover $\hat{f}(j)=0$ for $j<0$, then $f$ is called an $X$-valued analytic trigonometric polynomial.

Given $p \in[1: \infty)$ the Hardy space $H_{X}^{p}$ is defined to be the closure of all $X$ valued analytic trigonometric polynomials under the norm $\|\cdot\|_{p}$; equivalently,

$$
H_{X}^{p}=\left\{f \in L_{X}^{p}: \hat{f}(j)=0 \text { for } j<0\right\} .
$$

1.2. The spaces $H_{X}^{1}$, at and $\mathrm{BMO}_{X}$. Given a Banach space $X$, an $X$-atom supported on an interval $I \subset \mathbb{R}$ (with the Lebesgue measure $|I| \leq 2 \pi$ ) is any $X$-valued $2 \pi$-periodic function $a \in L_{X}^{\infty}$ such that

$$
\begin{gathered}
a(t)=0 \text { for } t \notin 2 k \pi+I \quad \text { for } k=0, \pm 1, \pm 2, \ldots, \\
\|a\|_{\infty} \leq 2 \pi|I|^{-1} ; \quad \int_{-\pi}^{\pi} a(t) d t=0 .
\end{gathered}
$$

Also every constant $X$-valued function $a(t)=x$ for $t \in \mathbb{R}$ with $\|x\| \leq 1$ is called an $X$-atom. Clearly, $\|a\|_{1} \leq 1$ for every $X$-atom $a$. If $a$ is an $X$-atom supported on $I$ then

$$
\|\hat{a}(j)\| \leq 4^{-1}|j||I| \text { for } j=0, \pm 1, \pm 2, \ldots .
$$

Indeed, if $t_{0}$ is the centre of $I$, then

$$
\begin{aligned}
2 \pi\|\hat{a}(j)\| & =\left\|\int_{I} a(t)\left(e^{-i j t}-e^{-i j t_{0}}\right) d t\right\| \leq\left\|a e^{-i j t_{0}}\right\|_{\infty} \int_{I}\left|e^{i j\left(t_{0}-t\right)}-1\right| d t \\
& \leq 2 \pi|I|^{-1} \int_{I}|j|\left|t-t_{0}\right| d t \leq 2 \pi|j||I|^{-1}\left(|I| 2^{-1}\right)^{2}=2 \pi|j||I| 4^{-1} .
\end{aligned}
$$

An $f \in L_{X}^{1}$ is said to have an atomic representation $\left(\left(a_{k}\right) \cdot\left(\lambda_{k}\right)\right)$ provided $\left(a_{k}\right)$ is a sequence of $X$-atoms, $\left(\lambda_{k}\right)$ is a sequence of nonnegative integers,

$$
\sum_{k} \lambda_{k}<+\infty ; \quad \lim _{n}\left\|f-\sum_{k=1}^{n} \lambda_{k} a_{k}\right\|_{1}=0
$$

We put

$$
\begin{aligned}
& H_{X}^{1, \text { at }}=\left\{f \in L_{X}^{1}: f \text { has an atomic representation }\right\}, \\
&\|f\|_{1, \text { at }}=\inf \sum \lambda_{k} \text { for } f \in H_{X}^{1, \text { at }}
\end{aligned}
$$

where the infimum is taken over all atomic representations of $f$. It is well known and easy to verify (cf. e.g. [GC-Ru] for the scalar case) that $H_{X}^{1, \text { at }}$ under the norm $\|\cdot\|_{1, \text { at }}$ is a Banach space; moreover, the natural inclusion $H_{X}^{1}$, at $\hookrightarrow L_{X}^{1}$ is a contraction: i.e.,

$$
\|f\|_{1} \leq\|f\|_{1, \text { at }} \text { for } f \in H_{X}^{1, \text { at }} .
$$

In general the norm $\|\cdot\|_{1}$, at is stronger than $\|\cdot\|_{1}$. However, on $H_{X}^{1}$ both norms are equivalent. Precisely we have 
Theorem 1.1 (Bourgain, Garcia-Cuerva). There is an absolute constant $C$ such that for every Banach space $X$

$$
\|f\|_{1, \text { at }} \leq C\|f\|_{1} \text { for } f \in H_{X}^{1} .
$$

Consequently every $f \in H_{X}^{1}$ belongs to $H_{X}^{1, \text { at }}$; the inclusion $H_{X}^{1} \hookrightarrow H_{X}^{1, \text { at }}$ is an isomorphic embedding of Banach spaces.

For the proof see [B1, Theorem 6].

Next we shall recall the definition $\mathrm{BMO}_{X}$. For $f \in L_{X}^{1}$ we put

$$
\|f\|_{\mathrm{BMO}}=\max \left(\|f\|_{1}, \sup (2 \pi|I|)^{-1} \int_{-\pi}^{\pi}\left\|f(t)-f_{I}\right\| d t\right)
$$

where $f_{I}=|I|^{-1} \int_{I} f(t) d t$ and the supremum is taken over all intervals $I \in \mathbb{R}$ with $|I| \leq 2 \pi$.

We define

$$
\mathrm{BMO}_{X}=\left\{f \in L_{X}^{1}:\|f\|_{\mathrm{BMO}}<+\infty\right\} .
$$

Clearly $\mathrm{BMO}_{X}$ under the norm $\|\cdot\|_{\mathrm{BMO}}$ is a Banach space.

Part of the Fefferman-Stein duality between $H^{1 \text {, at }}$ and BMO goes over the vector-valued case. To formulate the result it is convenient to introduce the following notation. Let $f \in L_{X}^{1}$ and $f^{*} \in L_{X^{*}}^{1}$ where $X^{*}$ is the dual of $X$. Write

$$
\left\langle f, f^{*}\right\rangle=(2 \pi)^{-1} \int_{-\pi}^{\pi}\left[f^{*}(t)\right](f(-t)) d t
$$

whenever the scalar-valued function $t \rightarrow\left[f^{*}(t)\right](f(-t))$ is integrable on $[-\pi, \pi]$. Theorem 1.2. For every Banach space $X$ the space $\mathrm{BMO}_{X^{*}}$ is isomorphic to a subspace of the dual of $H_{X}^{1}$, at via the map $f^{*} \rightarrow \phi_{f^{*}}$ where for $f^{*} \in \mathrm{BMO}_{X^{*}}$ the functional $\phi_{X^{*}} \in\left(H_{X}^{1, \text { at }}\right)^{*}$ is uniquely defined by its action on $X$-atoms, by $\phi_{f^{*}}(a)=\left\langle a, f^{*}\right\rangle$.

The proof is essentially the same as in the scalar case; it uses the fact that for every Banach space $X$ there is a natural isomorphic embedding of $L_{X^{*}}^{1}$ into $\left(L_{X}^{\infty}\right)^{*}$.

1.3. Some facts from geometry of Banach spaces. The classes of Banach spaces we will study have the property that if a space $X$ belongs to one of the classes, then every space finitely representable in $X$ also belongs to the same class. Recall that a Banach space $Y$ is crudely finitely representable in a Banach space $X$ if there is $K \geq 1$ such that for every finite-dimensional subspace $E$ of $Y$ there is a linear operator $u: E \rightarrow X$ such that $\|e\| \leq\|u(e)\| \leq K\|e\|$ for $e \in E$. As an example consider for $1 \leq p<\infty$ the space $H_{X}^{p}(D)$ of all $X$ valued analytic functions on the unit disc $D=\{z \in \mathbb{C}:|z|<1\}$ such that for each $0<r<1$ the function $F_{r} \in H_{X}^{P}$ and $\|F\|_{p}=\sup \left\{\left\|F_{r}\right\|_{p}: 0<r<1\right\}<$ $+\infty$ where $F_{r}(t)=F\left(r e^{i t}\right)$. Clearly $H_{X}^{P}$ isometrically embeds into $H_{X}^{P}(D)$. 
Conversely it is not hard to verify that $H_{X}^{P}(D)$ is crudely finitely representable in $H_{X}^{P}$.

Next recall (cf. [M-Pi]) that a Banach space $X$ has type $p$ (respectively cotype $q$ ) if there is a constant $T<\infty$ (respectively $C<\infty$ ) such that for every positive integer $m$ and every $x_{1}, x_{2}, \ldots, x_{m} \in X$

$$
\begin{aligned}
\int_{0}^{1}\left\|\sum_{k=1}^{m} x_{k} r_{k}(t)\right\| d t \leq T & \left(\sum_{k=1}^{m}\left\|x_{k}\right\|^{p}\right)^{1 / p} \\
& \left(\operatorname{resp} .\left(\sum_{k=1}^{m}\left\|x_{k}\right\|^{q}\right)^{1 / q} \leq C \int_{0}^{1}\left\|\sum_{k=1}^{m} x_{k} r_{k}(t)\right\| d t\right)
\end{aligned}
$$

where $\left(r_{k}\right)$ is the sequence of Rademacher functions. Type is a number in the interval $[1,2]$, while the range of cotype is the interval $[2,+\infty]$. A space is said to have nontrivial type (respectively nontrivial cotype) provided it has type $>1$ (respectively cotype $<+\infty)$. Spaces with type $>1$ are also called $B$-convex.

Note that the concepts of type and cotype do not change if in the definition the $L^{1}$-norm is replaced by any $L^{p}$-(quasi) norm for $0<p<\infty$ (the Kahane inequality [Kh, L-T]), and the Rademacher functions are replaced by an arbitrary sequence of distinct characters of a compact abelian group belonging to some Sidon set (Pisier [Pi1], cf. also [P]).

Following Peetre [Pee] we say that a Banach space $X$ has Fourier type $p$, where $1 \leq p \leq 2$, if there is a constant $K=K(X)$ such that

$$
\left(\sum_{-\infty}^{+\infty}\|\hat{f}(n)\|^{p^{\prime}}\right)^{1 / p^{\prime}} \leq K\|f\|_{p} \quad \text { for } f \in L_{X}^{p}
$$

where $p^{\prime}=p(p-1)^{-1}$ for $p>1$ and $p^{\prime}=\infty$ for $p=1$. A Banach space is said to have nontrivial Fourier type if it has Fourier type $p$ for some $p>1$.

1.4. $\left(H^{p}-l^{q}\right)$-multipliers and induced operators for vector-valued functions. Let $m=\left(m_{j}\right)_{j \geq 0}$ be a complex sequence and let $X$ be a Banach space. Define the operator $m_{x}$ from $X$-valued analytic trigonometric polynomials into the eventually zero $X$-valued sequences by $m_{X}(f)=\left(m_{j} \hat{f}(j)\right)_{j \geq 0}$. We call $m_{x}$ the operator induced by the multiplier $m$. The operator $m_{X}$ is said to be $(p, q)$ bounded provided there exists a constant $K=K(m, X)$ such that for every $X$-valued analytic trigonometric polynomial $f$

$$
\left(\sum_{j=0}^{\infty}\left\|m_{j} \hat{f}(j)\right\|^{q}\right)^{1 / q} \leq K\|f\|_{p} .
$$

If $m_{X}$ is $(p, q)$-bounded, then it uniquely extends to an operator (also denoted by $m_{X}$ ) from $H_{X}^{p}$ into the Banach space $l_{X}^{q}$ where

$$
l_{X}^{q}=\left\{\left(x_{j}\right) \subset X:\left\|\left(x_{j}\right)\right\|_{q}=\left(\sum\left\|x_{j}\right\|^{q}\right)^{1 / q}<\infty\right\} .
$$


We call $m$ an $\left(H^{p}-l^{q}\right)$-multiplier if for $X=\mathbb{C} m_{\mathbb{C}}$ is $(p, q)$-bounded.

Clearly, if $m \in l^{1}=l_{\mathbb{C}}^{1}$, then for every Banach space $X$ the induced operator $m_{X}$ is $(1,1)$-bounded. We shall show that for $X=c_{0}$ and for $X=L^{1} / \bar{H}_{0}^{1}$ if $m_{X}$ is $(1,1)$-bounded, then $m \in l^{1}$. Here $L^{1} / \bar{H}_{0}^{1}$ denotes the quotient of $L^{1}$ by the subspace

$$
\bar{H}_{0}^{1}=\left\{f \in L_{1}: \hat{f}(j)=0 \text { for } j \geq 0\right\} .
$$

In fact our result is slightly stronger.

Proposition 1.1. Let $X$ be either $c_{0}$ or $L^{1} / \bar{H}_{0}^{1}$. Assume that for some $m=\left(m_{j}\right)$ the operator $m_{x}$ is $(\infty, 1)$-bounded. Then $m \in l^{1}$.

Proof. $1^{\circ} . X=c_{0}$. Put $f_{n}=\sum_{j=0}^{n} e^{i j t} \delta_{j}$ for $n=1,2, \ldots$, where $\left(\delta_{j}\right)_{j \geq 0}$ is the unit vector basis of $c_{0}$. Clearly, for all $n,\left\|f_{n}\right\|_{\infty}=1$ and

$$
\sum_{j=0}\left\|m_{j} \hat{f}_{n}(j)\right\|=\sum_{j=0}^{\infty}\left|m_{j}\right| \leq K<\infty .
$$

$2^{\circ}$. $X=L^{1} / \bar{H}_{0}^{1}$. Let $F^{(n)}$ denote the $n$th Fejér kernel. Consider the $L^{1}$ valued trigonometric polynomial $t \rightarrow F_{t}^{(n)}$ where $F_{t}^{(n)}(s)=F^{(n)}(s+t)$. Put $f_{n}(t)=q\left(F_{t}^{(n)}\right)$ where $q: L^{1} \rightarrow L^{1} / \bar{H}_{0}^{1}$ is the quotient map. If $j=0, \pm 1$, $\pm 2, \ldots$ then

$\hat{f}_{n}(j)=(2 \pi)^{-1} \int_{-\pi}^{\pi} q\left(F_{t}^{(n)}\right) e^{-i j t} d t=q\left((2 \pi)^{-1} \int_{-\pi}^{\pi} F_{t}^{(n)} e^{-i j t} d t\right)=\hat{F}^{(n)}(j) q\left(e_{j}\right)$, where $e_{j}(s)=e^{i j s}$ for $s \in \mathbb{R}$. Thus $f_{n}$ is an $L^{1} / \bar{H}_{0}^{-1}$-valued analytic trigonometric polynomial because $q\left(e_{j}\right)=0$ for $j<0$. Clearly

$$
\left\|f_{n}(t)\right\| \leq\|q\|\left\|F_{t}^{(n)}\right\|_{L^{1}}=1 \quad \text { for } t \in \mathbb{R}
$$

because $\left\|F_{t}^{(n)}\right\|_{L^{1}}=\left\|F^{(n)}\right\|_{L^{1}}=1$ and $\|q\|=1$. Next note that $\left\|q\left(e_{j}\right)\right\|=1$ for $j \geq 0$. Indeed, $\left\|q\left(e_{j}\right)\right\| \leq\left\|e_{j}\right\|_{L^{1}} \leq 1$. On the other hand,

$$
\begin{aligned}
\left\|q\left(e_{j}\right)\right\| & =\inf \left\{(2 \pi)^{-1} \int_{-\pi}^{\pi}\left|e_{j}(s)-g(s)\right| d s: g \in \bar{H}_{0}^{1}\right\} \\
& \geq \inf \left\{(2 \pi)^{-1}\left|\int_{-\pi}^{\pi}\left(1-e_{-j}(s) g(s)\right) d s\right|: g \in \bar{H}_{0}^{1}\right\} \geq 1,
\end{aligned}
$$

because $\int_{-\pi}^{\pi} e_{-j}(s) g(s) d s=0$ for $j \geq 0$ and for $g \in \bar{H}_{0}^{1}$. Thus, for $n=$ $1,2, \ldots$,

$$
\sum_{j=0}^{\infty}\left\|m_{j} \hat{f}_{n}(j)\right\|=\sum_{j=0}^{\infty}\left|m_{j}\right|\left|\widehat{F}^{(n)}(j)\right| \leq K .
$$

Hence, $\sum_{j=0}^{\infty}\left|m_{j}\right| \leq K<\infty$, because $\lim _{n} \widehat{F}^{(n)}(j)=1$, for all $j$.

Remark. Proposition 1.1 remains valid for every Banach space $E$ in which either $c_{0}$ or $L^{1} / \bar{H}_{0}^{1}$ is crudely finitely representable. In particular one can take 
$E$ as any infinite-dimensional $\mathscr{L}_{\infty}$-space or $C^{*}$-algebra as well as the reflexive space $\left(l_{2}^{\infty} \times l_{3}^{\infty} \times \cdots\right)_{l_{2}}$.

\section{BANACH SPACES CHARACTERIZED BY $\left(H^{1}-l^{1}\right)$-MULTIPLIERS}

Definition 2.1. A Banach space $X$ is of $\left(H^{1}-l^{1}\right)$-Fourier type provided for every $\left(H^{1}-l^{1}\right)$-multiplier $m$ the induced multiplier $m_{x}$ is $(1,1)$-bounded. Recall the elegant description $\left(H^{1}-l^{1}\right)$-multipliers.

Theorem 2.1 (Ch. Fefferman). A scalar sequence $m=\left(m_{j}\right)_{j \geq 0}$ is an $\left(H^{1}-l^{1}\right)$ multiplier iff

$$
\rho(m)=\left(\left|m_{0}\right|^{2}+\left|m_{1}\right|^{2}+\sup _{s \geq 1} \sum_{k=1}^{\infty}\left(\sum_{j=k s+1}^{(k+1) s}\left|m_{j}\right|\right)^{2}\right)^{1 / 2}<\infty .
$$

For the proof see $[\mathrm{Sz}-\mathrm{W}]$.

In the sequel $F M$ stands for the Banach space of all scalar sequences satisfying (2.1) equipped with the norm $\rho(\cdot)$.

We begin with a dual description of $\left(H^{1}-l^{1}\right)$ Fourier type spaces.

Proposition 2.1. For every Banach space $X$ the following are equivalent:

(i) $X$ is an $\left(H^{1}-l^{1}\right)$-Fourier type space;

(ii) there is $C>0$ such that for every $m \in F M$ and $f \in H_{X}^{1}$

$$
\sum_{j: 0}\left\|m_{j} \hat{f}(j)\right\| \leq C \rho(m)\|f\|_{1} ;
$$

(iii) there is $C>0$ such that for every eventually zero sequence $\left(x_{j}^{*}\right)_{j \geq 0}$ of elements of $X^{*}$ there is an $X^{*}$-valued trigonometric polynomial $g^{*}$ such that

$$
\hat{g}^{*}(j)=x_{j}^{*} \quad \text { for } j \geq 0 ; \quad\left\|g^{*}\right\|_{\infty} \leq C \rho\left(\left(\left\|x_{j}^{*}\right\|\right)_{j \geq 0}\right) .
$$

Proof. (i) $\Rightarrow$ (ii). Put $\rho_{X}(m)=\sup \left\{\sum_{j \geq 0}\left\|m_{j} \hat{f}(j)\right\|: f \in H_{X}^{1} ;\|f\|_{1}=1\right\}$. The standard Baire category argument yields that $\rho_{X}(\cdot)$ is a bounded norm on $F M$. This yields (ii).

(ii) $\Rightarrow$ (iii). Let $x_{j}^{*}=0$ for $j \geq N$. Define on $H_{X}^{1}$ the linear functional $\phi_{0}^{*}$ by $\phi_{0}^{*}(f)=\sum_{j=0}^{N} x_{j}^{*}(\hat{f}(j))$ for $f \in H_{X}^{1}$. It follows from (ii) that $\left\|\phi_{0}^{*}\right\| \leq$ $C \rho\left(\left(\left\|x_{j}^{*}\right\|\right)_{j \geq 0}\right)$. Let $\phi^{*}$ be a norm-preserving extension of $\phi_{0}^{*}$ onto $L_{X}^{1}$. Let $V$ be the $N$ th de la Vallé Poussin kernel, i.e., $\widehat{V}(j)=1$ for $|j| \leq N, \widehat{V}(j)=0$ for $|j| \geq 2 N$ and $\widehat{V}(j)$ linear for $-2 N \leq j \leq-N$ and for $N \leq j \leq 2 N$. It is well known that $\|V\|_{1} \leq 2$. Define for $j=0, \pm 1, \pm 2, \ldots, y_{j}^{*} \in X^{*}$ by

$$
y_{j}^{*}(x)=\widehat{V}(j) \phi\left(x e_{j}\right) \quad \text { for } x \in X,
$$

where $e_{j}(t)=e^{i j t}$. Put $g^{*}(t)=\sum_{|j| \leq 2 N} y_{j}^{*} e^{i j t}$. Then (denoting by $a * b$ the convolution of the functions $a$ and $b$ )

$$
\left\langle f, g^{*}\right\rangle=\phi^{*}(V * f) \text { for } f \in L_{X}^{1} .
$$


Thus, remembering that $\|V\|_{1} \leq 2$, we get

$$
\left\|g^{*}\right\|_{\infty} \leq\left\|\phi^{*}\right\|\|V\|_{1} \leq 2 C \rho\left(\left(\left\|x_{j}^{*}\right\|\right)_{j \geq 0}\right) .
$$

On the other hand, taking into account that $x \cdot e_{j} \in H_{X}^{1}$ for $j \geq 0$ and $x \in X$, we get for $0 \leq j \leq N$

$$
\hat{g}^{*}(j)(x)=y_{j}^{*}(x)=\widehat{V}(j) \phi^{*}\left(x e_{j}\right)=\widehat{V}(j) \phi_{0}^{*}\left(x e_{j}\right)=x_{j}^{*}(x) .
$$

Thus $\hat{g}^{*}(j)=x_{j}^{*}$ for $0 \leq j \leq N$.

(iii) $\Rightarrow$ (i). Let $m \in F M$ and let $f$ be an $X$-valued analytic trigonometric polynomial of degree $N$. For $j=0,1, \ldots, N$ pick $y_{j}^{*} \in X$ so that $\left\|y_{j}^{*}\right\|=1$ and $y_{j}^{*}(\hat{f}(j))=\|\hat{f}(j)\|$. Put $x_{j}^{*}=\left|m_{j}\right| y_{j}^{*}$ for $0 \leq j \leq N$ and $x_{j}^{*}=0$ for $j>$ $N$. Obviously $\rho\left(\left(\left\|x_{j}^{*}\right\|_{j \geq 0}\right) \leq \rho(m)\right.$. By (iii) there is an $X$-valued trigonometric polynomial $g^{*}$ satisfying $(2.2)$. We have

$$
\begin{aligned}
\sum_{j=0}^{\infty}\left\|m_{j} \hat{f}(j)\right\| & =\left\langle f, g^{*}\right\rangle \leq\left\|g^{*}\right\|_{\infty}\|f\|_{1} \\
& \leq C \rho\left(\left(\left\|x_{j}^{*}\right\|\right)_{j \geq 0}\right)\|f\|_{1} \leq C \rho(m)\|f\|_{1} .
\end{aligned}
$$

Hence $m_{X}$ is $(1,1)$-bounded.

Clearly Definition 2.1 yields

Corollary 2.1. Every Banach space crudely finitely representable in a space of $\left(H^{1}-l^{1}\right)$-Fourier type is an $\left(H^{1}-l^{1}\right)$-Fourier type space.

Let $(\mu, \Omega)$ be a measure space and let $X$ be a Banach space. By $L_{X}^{1}(\mu)$ we denote the space of $X$-valued Bochner $\mu$ integrable functions on $\Omega$.

Proposition 2.2. If $X$ is an $\left(H^{1}-l^{1}\right)$-Fourier type space so is $L_{X}^{1}(\mu)$.

Proof. It is enough to show that $l_{X}^{1}$ is of $\left(H^{1}-l^{1}\right)$-Fourier type, because for every measure space $(\mu, \Omega), L_{X}^{1}(\mu)$ is finitely representable in $l_{X}^{1}$.

Let $f=\left(f_{k}\right)$ be an $l_{X}^{1}$-valued analytic trigonometric polynomial. Then obviously each of the coordinates $f_{k}$ is an $X$-valued analytic trigonometric polynomial. Hence, by the hypothesis on $X$ there is $C>0$, such that for every $m \in F M$

$$
\sum_{j=0}^{\infty}\left\|m_{j} \hat{f}_{k}(j)\right\|_{X} \leq C \rho(m)\left\|f_{k}\right\|_{X} \quad(k=0,1, \ldots) .
$$

Summing over all $k$, we get

$$
\begin{aligned}
\sum_{j=0}^{\infty}\left\|m_{j} \hat{f}(j)\right\|_{l^{\prime}} & =\sum_{j=0}^{\infty} \sum_{k=0}^{\infty}\left\|m_{j} \hat{f}_{k}(j)\right\|_{X} \\
& \leq \sum_{k=0}^{\infty} C \rho(m)\left\|f_{k}\right\|_{X}=C \rho(m)\|f\|_{l_{Y}^{\prime}} .
\end{aligned}
$$


Corollary 2.2. If $1 \leq p \leq 2$, then $L^{p}$ is an $\left(H^{1}-l^{1}\right)$-Fourier type space.

Proof. By Proposition 2.2, $L^{1}=L_{\mathbb{C}}^{1}$ is of $\left(H^{1}-l^{1}\right)$-Fourier type. If $1 \leq p \leq 2$ then $L^{p}$ is isometrically isomorphic to a subspace of $L^{1}$ (cf. e.g. [R] for the complex case).

Another obvious but useful consequence of Definition 2.1 is

Corollary 2.3. Assume that a Banach space $X$ satisfies

(*) there is $C>0$ such that for every $f \in H_{X}^{1}$ there is a complex valued function $\phi \in H^{1}$ such that

$$
\|\hat{f}(j)\| \leq|\hat{\phi}(j)| \quad \text { for } j=0,1, \ldots ; \quad\|\phi\|_{1} \leq C\|f\|_{1} .
$$

Then $X$ is an $\left(H^{1}-l^{1}\right)$-Fourier type space.

For $1 \leq p \leq \infty$ let $S_{p}$ denote the $p$ th Schatten-von Neumann unitary ideal, i.e. the space of all operators $A: l^{2} \rightarrow l^{2}$ such that

$$
\|A\|_{S_{p}}=\left(\operatorname{trace}\left(A^{*} A\right)^{p / 2}\right)^{1 / p}<\infty .
$$

It is well known (cf. [G-K]) that $S_{1}$ coincides with the space of all nuclear operators on $l^{2}$ and $S_{2}$ with the space of all Hilbert-Schmidt operators.

Theorem 2.2. (a) The space $S_{1}$ satisfies $(*)$ with $C=1$, hence it is an $\left(H^{1}-l^{1}\right)$ Fourier type space

(b) If $1 \leq p \leq 2$ then $S_{p}$ is an $\left(H^{1}-l^{2}\right)$-Fourier type space.

The proof of Theorem 2.2 is based upon

Theorem 2.3 (the noncommutative factorization theorem). For every $f \in H_{S_{p}}^{1}$ there are $g$ and $h$ in $H_{S_{2 p}}^{2}$ such that

$$
f=g \circ h, \quad\|f\|_{S_{p}, 1}=\|g\|_{S_{2 p}, 2}\|h\|_{S_{2 p}, 2} .
$$

Here $f=g \circ h$ means $f(t)=g(t) \circ h(t)$ for $t \in \mathbb{R}$, i.e. at each point $t$ the operator $f(t)$ is the composition of the operator $h(t)$ with the operator $g(t)$. To avoid misunderstanding we write here

$$
\|f\|_{S_{p}, r}=\left((2 \pi)^{-1} \int_{-\pi}^{\pi}\|f(t)\|_{S_{p}}^{r} d t\right)^{1 / r} \text { for } 1 \leq p<\infty \text { and } 1 \leq r<\infty .
$$

Theorem 2.3 is due to combined efforts of Lowdenslager, Helson, Devinatz, Douglas, and Sarason (cf. [S, §10] and references there).

Proof of Theorem 2.2. Let $f \in H_{S_{1}}^{1}$ and $g, h \in H_{S_{2}}^{2}$ satisfy (2.4). Put

$$
\phi(t)=\sum_{j=0}^{\infty} \sum_{k=0}^{j}\|\hat{g}(k)\|_{S_{2}}\|\hat{h}(j-k)\|_{S_{2}} e^{i j t} .
$$


Using the inequality $\|A \circ B\|_{S_{1}} \leq\|A\|_{S_{2}}\|B\|_{S_{2}}$ and (2.4) we get

$$
|\hat{\phi}(j)| \geq \sum_{k=0}^{j}\|\hat{g}(k) \circ \hat{h}(j-k)\|_{S_{1}} \geq\left\|\sum_{k=0}^{j} \hat{g}(k) \circ \hat{h}(j-k)\right\|_{S_{1}}=\|\hat{f}(j)\|_{S_{1}} .
$$

On the other hand, note that $\phi=G \cdot H$ where

$$
G=\sum_{j=0}^{\infty}\|\hat{g}(j)\|_{S_{2}} e^{i j t}, \quad H=\sum_{j=0}^{\infty}\|\hat{h}(j)\|_{S_{2}} e^{i j t}
$$

Next observe that if $E$ is a Hilbert space, then for every $F \in H_{E}^{2},\|F\|_{2}^{2}=$ $\sum_{j=0}^{\infty}\|\widehat{F}(j)\|_{2}^{2}$ and conversely for every sequence $\left(x_{j}\right) \in E$ with $\sum\left\|x_{j}\right\|_{E}^{2}<$ $\infty$ the function $\sum_{j \geq 0} x_{j} e^{i j t}$ belongs to $H_{E}^{2}$. Thus remembering that $S_{2}$ is a Hilbert space we get $\|G\|_{2}=\|g\|_{S_{2}, 2}$ and $\|H\|_{2}=\|h\|_{S_{2}, 2}$. Now using the Schwarz inequality and (2.4) we get

$$
\|\phi\|_{1} \leq\|G\|_{2}\|H\|_{2}=\|g\|_{S_{2}, 2}\|h\|_{S_{2}, 2}=\|f\|_{S_{1}, 1} .
$$

This proves part (a).

To prove part (b), fix $m \in F M$ and define for $\nu=1,2$ the bilinear operators $U_{\nu}: L_{S_{2 \nu}}^{2} \times L_{S_{2 \nu}}^{2} \rightarrow l_{S_{\nu}}^{1}$ by

$$
U_{\nu}(g, h)=\left(m_{j} \sum_{k=0}^{j} \hat{g}(k) \hat{h}(j-k)\right) .
$$

First we verify that $U_{\nu}$ is bounded for $\nu=1,2$. Since $S_{2}$ is a Hilbert space and $S_{4}$ is a UMD space (cf. [B1, Be-Gi-Mu]) there are $K_{\nu}>0$ for $\nu=1,2$ (actually $K_{1}=1$ ), such that if $f \in L_{S_{2 \nu}}^{2}$ then $R f \in H_{S_{2 \nu}}^{2}$ and $\|R f\|_{S_{2 \nu, 2}} \leq K_{\nu}\|f\|_{S_{2 \nu, 2}}$ where $R f$ is defined by $(R f)^{\wedge}(j)=\hat{f}(j)$ for $j \geq 0$ and $(R f)^{\wedge}(j)=0$ for $j<0$. Next observe that if $g$ and $h$ are in $L_{S_{2 \nu}}^{2}$ then $f=R g \circ R h \in H_{S_{\nu}}^{1}$. Indeed, using the inequality $\|A \circ B\|_{S_{p}} \leq\|A\|_{S_{2 p}}\|B\|_{S_{2 p}}$ and Schwarz's inequality we get

$$
\begin{aligned}
\|f\|_{S_{\nu}, 1} & \leq(2 \pi)^{-1} \int_{-\pi}^{\pi}\|(R g)(t)\|_{S_{2 \nu}} \cdot\|(R h)(t)\|_{S_{2 \nu}} d t \\
& \leq\|R g\|_{S_{2 \nu, 2}}\|R h\|_{S_{2 \nu, 2}} \leq K_{\nu}^{2}\|g\|_{S_{2 \nu, 2}}\|h\|_{S_{2 \nu, 2}} .
\end{aligned}
$$

Now, by part (a) of the theorem, $S_{1}$ is an $\left(H^{1}-l^{1}\right)$-Fourier type space; $S_{2}$ being a Hilbert space has also the same property. Thus there are constants $C_{\nu}>0$ for $\nu=1,2$ such that

$$
\sum\left\|m_{j} \hat{f}(j)\right\|_{S_{\nu}} \leq C_{\nu} \rho(m)\|f\|_{S_{\nu}, 1} \quad \text { for every } f \in H_{S_{\nu}}^{1}
$$


In particular for $f=R g \circ R h$ we have

$$
\begin{aligned}
\left\|U_{\nu}(g, h)\right\|_{l_{S_{\nu}}} & =\sum_{j \geq 0}\left\|m_{j} \sum_{k=0}^{j} \hat{g}(k) \hat{h}(j-k)\right\|_{S_{\nu}} \\
& =\sum_{j \geq 0}\left\|m_{j} \sum_{k=0}^{j}(R g) \hat{(k)(R h)}(j-k)\right\|_{S_{\nu}} \\
& =\sum_{j \geq 0}\left\|m_{j} \hat{f}(j)\right\| \leq C_{\nu} K_{\nu}^{2} \rho(m)\|g\|_{S_{2 \nu, 2}}\|h\|_{S_{2 \nu, 2}} .
\end{aligned}
$$

This proves the boundedness of the operators $U_{1}$ and $U_{2}$.

Note that $L_{S_{2 p}}^{2} \times L_{S_{2 p}}^{2}$ and $l_{2 p}^{1}$ are for $1 \leq p \leq 2$ interpolation scales (cf. [BG-Lö]). Thus, by an interpolation theorem for multilinear operators (cf. [BgLö], p. 96) we infer that for each $p$ with $1 \leq p \leq 2$ there is a constant $M_{p}>0$, such that $\left\|U_{p}(g, h)\right\|_{l_{S_{p}}^{1}} \leq M_{p}\|g\|_{S_{2 p, 2}}\|h\|_{2 p, 2}$ for $g \in L_{S_{2 p}}^{2}, h \in L_{S_{2 p}}^{2}$, where $U_{p}: L_{S_{2 p}}^{2} \times L_{S_{2 p}}^{2} \rightarrow l_{S_{p}}^{1}$ is defined by $U_{p}(g, h)=\left(m_{j} \sum_{k=0}^{j} \hat{g}(k) \circ \hat{h}(j-k)\right)_{j \geq 0}$.

Now let $f \in H_{S_{p}}^{1}$. By Theorem 2.3 there are $g \in H_{S_{2 p}}^{2}$ and $h \in H_{S_{2 p}}^{2}$ so that (2.4) is satisfied. Thus we have

$$
\sum_{j \geq 0}\left\|m_{j} \hat{f}(j)\right\|_{S_{p}}=\left\|U_{p}(g, h)\right\|_{l_{S_{p}}^{\prime}} \leq M_{p}\|g\|_{S_{2 p}, 2} \leq M_{p}\|f\|_{S_{1}, 1} .
$$

Corollary 2.4. The dual of $B\left(l^{2}\right)$ has $\left(H^{1}-l^{1}\right)$-Fourier type.

Proof. We have $S_{1}^{*}=B\left(l^{2}\right)$. Hence $\left[B\left(l^{2}\right)\right]^{*}$ is finitely representable in $S_{1}$ because by the Local Reflexivity Principle (cf. [L-R]) the second dual of any Banach space is finitely representable in the space.

Remarks. $1^{\circ}$. Clearly $X$ has $(*)$ if there is $C>0$, such that for every $X$ valued analytic trigonometric polynomial there is $\phi \in H^{1}$, such that (2.4) holds. Thus if $X$ has $(*)$, so does every space crudely finitely representable in $X$.

$2^{\circ}$. The classical factorization theorem for $H^{1}$-functions easily yields that $l^{1}$ has $(*)$. Indeed given $f=\left(f_{k}\right) \in H_{l^{1}}^{1}$, pick by the factorization theorem $g=\left(g_{k}\right)$ and $h=\left(h_{k}\right)$ so that $g_{k} \in H^{2}, h_{k} \in H^{2}$ and $\left\|g_{k}\right\|_{2}=\left\|h_{k}\right\|_{2}=\left\|f_{k}\right\|_{1}^{1 / 2}$ for $k=0,1,2, \ldots$. Then $g \in H_{l^{2}}^{2} ; h \in H_{l^{2}}^{2}, f=g h$ and $\|f\|_{1}=\|g\|_{2}\|h\|_{2}$. The rest of the argument is the same as in the proof of Theorem 2.2.

$3^{\circ}$. For every measure space $(\Omega, \mu)$ the space $L^{1}(\mu)$ is finitely representable in $l^{1}$. Thus, for $1 \leq p \leq 2, L^{p}(\mu)$ has $(*)$.

Next we discuss some other classes of $\left(H^{1}-l^{1}\right)$-multipliers and related classes of Banach spaces.

Let $1 \leq p \leq 2$. Denote by $F M_{p}$ the Banach space (under the norm $\rho_{p}(\cdot)$ ) 
of scalar sequences $m=\left(m_{j}\right)_{j \geq 0}$, such that

$$
\rho_{p}(m)=\left(\sup _{s>1} \sum_{k=1}^{\infty}\left(\sum_{k s+1}^{(k+1) s}\left|m_{j}\right|\right)^{p}+\left|m_{1}\right|^{p}+\left|m_{0}\right|^{p}\right)^{1 / p}<\infty .
$$

Observe that

$$
\begin{gathered}
F M_{2}=F M ; \quad F M_{1}=l^{1} ; \quad \text { if } 1 \leq p_{1} \leq p_{2} \text { then } F M_{p_{1}} \subset F M_{p_{2}}, \\
\left((j+1)^{-1}\right)_{j \geq 0} \in \bigcap_{p>1} F M_{p} .
\end{gathered}
$$

Definition 2.2. A Banach space $X \in \mathscr{F}_{p}$ if for every multiplier $m \in F M_{p}$ the induced operator $m_{X}$ is $(1,1)$-bounded.

A Banach space $X$ is an $\mathscr{H} \mathscr{F}$ space, in symbols $X \in \mathscr{H} \mathscr{F}$ if there is $C>0$, such that

$$
\sum_{j>0}^{\infty}(j+1)^{-1}\|\hat{f}(j)\| \leq C\|f\|_{1} \quad \text { for } f \in H_{X}^{1} .
$$

$\mathscr{H} \mathcal{F}$ stands for "Hardy inequality"; obviously (2.7) is a vector-valued analogue of the Hardy inequality [Du].

Note that inclusions $(2.6)$ yield that $\mathscr{F}_{2}$ is the class of all spaces of $\left(H^{1}-l^{1}\right)$ Fourier type. $\mathscr{F}_{1}$ is the class of all Banach spaces (by the observation before Proposition 1.1) and

$$
\text { if } 1 \leq p_{1} \leq p_{2} \leq 2 \text {, then } \mathscr{F}_{p_{1}} \supset \mathscr{F}_{p_{2}}, \quad \mathscr{F}_{1} \supset \mathscr{H} \mathscr{F} \supset \bigcup_{p>1} \mathscr{F}_{p} \text {. }
$$

Proposition 2.1 generalizes to the case of $\mathscr{F}_{p}$; we replace in (ii) and (iii) $\rho(m)$ by $\rho_{p}(m)$. The proof remains the same. We also have a similar result for $\mathscr{H} \mathscr{S}$ spaces.

Proposition 2.3. For every Banach space $X$ the following are equivalent:

(j) $X \in \mathscr{H} \mathcal{F}$,

(jj) There is $C>0$ such that for every eventually zero sequence $\left(x_{j}^{*}\right)_{j \geq 0}$ in $X^{*}$ with $\left\|x_{j}^{*}\right\| \leq(j+1)^{-1}$ for $j=0,1, \ldots$ there is an $X^{*}$-valued trigonometric polynomial $g^{*}$ such that $\hat{g}^{*}(j)=x_{j}^{*}$ for $j=0,1,2, \ldots$ and $\left\|g^{*}\right\|_{\infty} \leq C$.

Next we examine connections between Fourier type, type, and $\mathscr{F}_{p}$ classes.

First we have

Theorem 2.4. If a Banach space $X$ has Fourier type $p$ then $X \in \mathscr{F}_{p}$.

Proof. By Theorem 1.1 it suffices to show that there is $C>0$ such that for every $X$-atom $a$ and every $m \in F M_{p}$

$$
\Sigma=\sum_{j=0}^{\infty}\left\|m_{j} \hat{a}(j)\right\| \leq C \rho_{p}(m) .
$$


This is trivial for constant atoms. Otherwise it is enough to consider atoms supported on an interval $[-\delta, \delta]$ for some $\delta$ with $0<\delta<\pi$ (because a translate of an atom is again an atom). Given $\delta$ as above pick a positive integer $s$ so that $s \delta \leq 1<s \delta+\delta$. Put

$$
\Sigma_{1}=\sum_{j=0}^{s}\left\|m_{j} \hat{a}(j)\right\| ; \quad \Sigma_{2}=\sum_{j=s+1}^{\infty}\left\|m_{j} \hat{a}(j)\right\|=\sum_{k=1}^{\infty} \sum_{k s+1}^{(k+1) s}\left\|m_{j} \hat{a}_{j}\right\| .
$$

To estimate $\Sigma_{1}$ note that if $m \in F M_{p}$ then

$$
\sum_{2^{r-1}+1}^{2^{r}}\left|m_{j}\right|=\sum_{2^{r-1} 1+1}^{2^{r-1}(1+1)}\left|m_{j}\right| \leq \rho_{p}(m) \text { for } r=1,2, \ldots
$$

Pick a nonnegative integer $r_{0}$ so that $2^{r_{0}} \leq s<2^{r_{0}+1}$. Then $\delta 2^{r_{0}+1}<2 s \delta \leq 2$. Thus remembering that (by 1.1) $\|\hat{a}(j)\| \leq \delta j 8^{-1}$ for $j=0,1, \ldots$ we get

$$
\begin{aligned}
\Sigma_{1} & \leq \sum_{j=0}^{2^{r_{0}+1}}\left\|m_{j} \hat{a}(j)\right\| \leq\left\|m_{0} \hat{a}(0)\right\|+\left\|m_{1} \hat{a}(1)\right\|+\sum_{r=1}^{r_{0}+1} \sum_{2^{r-1}+1}^{2^{r}}\left\|m_{j} \hat{a}(j)\right\| \\
& \leq 8^{-1} \delta\left(\left|m_{1}\right|+\sum_{r=1}^{r_{0}+1} \sum_{2^{r-1}+1}^{2^{r}} j\left|m_{j}\right|\right) \\
& \leq 8^{-1} \delta \rho_{p}(m)\left(1+\sum_{r=1}^{r_{0}+1} 2^{r}\right) \leq 2^{-1} \rho_{p}(m) .
\end{aligned}
$$

To estimate $\Sigma_{2}$ for $k=1,2 \ldots$ pick $j_{k}$ so that

$$
\left\|\hat{a}\left(j_{k}\right)\right\|=\max \{\|\hat{a}(j)\|: k s<j \leq(k+1) s\} .
$$

Then, by the Hölder inequality, we get

$$
\begin{aligned}
\Sigma_{2} & \leq \sum_{k=1}^{\infty}\left\|\hat{a}\left(j_{k}\right)\right\| \sum_{j=k s+1}^{(k+1) s}\left|m_{j}\right| \\
& \leq T^{1 / p^{\prime}}\left(\sum_{k=1}^{\infty}\left(\sum_{j=k s+1}^{(k+1) s}\left|m_{j}\right|\right)^{p}\right)^{1 / p} \leq \rho_{p}(m) T^{1 / p^{\prime}}
\end{aligned}
$$

where $T=\sum_{k=1}^{\infty}\left\|\hat{a}\left(j_{k}\right)\right\|^{p^{\prime}}$. To estimate $T$ first observe that for fixed $k=$ $1,2, \ldots$ and $r=1,2, \ldots, s$ we have

$$
\begin{aligned}
\left\|\hat{a}\left(j_{k}\right)\right\| & \leq\|\hat{a}(k s+r)\|+\sum_{j=k s+1}^{(k+1) s}\|\hat{a}(j)-\hat{a}(j+1)\| \\
& \leq\|\hat{a}(k s+r)\|+s^{1 / p}\left(\sum_{j=k s+1}^{(k+1) s}\|\hat{a}(j)-\hat{a}(j+1)\|^{p^{\prime}}\right)^{1 / p^{\prime}} .
\end{aligned}
$$


Thus using again the Hölder inequality

$$
\left\|\hat{a}\left(j_{k}\right)\right\|^{p^{\prime}} \leq 2^{p^{\prime} / p}\left(\|\hat{a}(k s+r)\|^{p^{\prime}}+s^{p^{p^{\prime}} / p} \sum_{j=k s+1}^{(k+1) s}\|\hat{a}(j)-\hat{a}(j+1)\|^{p^{\prime}}\right) .
$$

Averaging over $r=1,2, \ldots, s$ we obtain

$$
\left\|\hat{a}\left(j_{k}\right)\right\|^{p^{\prime}} \leq 2^{p^{\prime} / p}\left(s^{-1} \sum_{j=k s+1}^{(k+1) s}\|\hat{a}(j)\|^{p^{\prime}}+s^{p^{\prime} / p} \sum_{j=k s+1}^{(k+1) s}\|\hat{a}(j)-\hat{a}(j+1)\|^{p^{\prime}}\right) .
$$

Thus summing over $k=1,2 \ldots$ we get

$$
\begin{aligned}
T & \leq 2^{p^{p^{\prime}} / p}\left(s^{-1} \sum_{j=s+1}^{\infty}\|\hat{a}(j)\|^{p^{\prime}}+s^{p^{\prime} / p} \sum_{j=s+1}^{\infty}\|\hat{a}(j)-\hat{a}(j+1)\|^{p^{\prime}}\right) \\
& =2^{p^{p^{\prime}} / p}\left(s^{-1} \sum_{j=s+1}^{\infty}\|a(j)\|^{p^{\prime}}+s^{p^{\prime} / p} \sum_{j=s+1}^{\infty}\|\hat{d}(j)\|^{p^{\prime}}\right),
\end{aligned}
$$

where $d(t)=a(t)\left(1-e^{-i t}\right)$.

Now use the hypothesis that $X$ has Fourier type $p$, say with constant $K$. Then

$$
T \leq 2^{p^{\prime} / p} K^{p^{\prime}}\left(s^{-1}\|a\|_{p}^{p^{\prime}}+s^{p^{\prime} / p}\|d\|_{p}^{p^{\prime}}\right) .
$$

It follows from the definition of an $X$-atom supported on $[-\delta, \delta]$ that $\|a\|_{p} \leq$ $\|a\|_{1}^{1 / p}\|a\|_{\infty}^{1 / p^{\prime}} \leq\left(\pi \delta^{-1}\right)^{1 / p^{\prime}}$. Hence

$$
\|d\|_{p} \leq \sup _{|t| \leq \delta}\left|1-e^{-i t}\right|\left(\pi \delta^{-1}\right)^{1 / p^{\prime}} \leq \pi^{1 / p^{\prime}} \delta^{1-1 / p^{\prime}} .
$$

Thus

$$
T \leq 2^{p^{\prime} / p} \pi K^{p^{\prime}}\left(s^{-1} \delta^{-1}+s^{p^{\prime} / p} \delta^{p^{\prime} / p}\right) \leq 2^{p^{\prime} / p} \pi K^{p^{\prime}}\left(1+s^{-1}+1\right)<6 \pi K^{p^{\prime}} .
$$

Therefore $\Sigma_{2} \leq(6 \pi)^{p^{\prime} / p} K \rho_{p}(m)$, and consequently $\Sigma \leq\left(2^{-1}+(6 \pi)^{p^{\prime} / p} K\right) \rho_{p}(m)$.

Corollary 2.5. If $X$ has nontrivial Fourier type then $X \in \mathscr{H} \mathscr{S}$.

Next we have

Proposition 2.4. If $X \in \mathscr{F}_{p}$ for some $p$ with $1 \leq p \leq 2$ then $X$ has cotype $p^{\prime}$; in particular all $\left(H^{1}-l^{1}\right)$-Fourier type spaces have cotype 2 .

Proof. For arbitrary scalar sequence $\left(\lambda_{r}\right)_{r \geq 1}$ with $\sum_{r=1}^{\infty}\left|\lambda_{r}\right|^{p}=1$ define $m=$ $\left(m_{j}\right)_{j \geq 0}$ by $m_{j}=0$ for $j \neq 2,4,8, \ldots ; m_{2^{r}}=\lambda_{r}$ for $r=1,2, \ldots$ Then a direct computation shows that $\rho_{p}(m)=1$. Thus if $X \in \mathscr{F}_{p}$ and $\left(x_{r}\right)_{r \geq 1}$ is any eventually zero sequence in $X$ then for the $X$-valued analytic trigonometric polynomial $f=\sum_{r \geq 1} x_{r} e^{2^{r} t i}$ we have the estimate

$$
\sum_{r \geq 1}\left\|\lambda_{r} x_{r}\right\| \leq C\|f\|_{1} \text { for }\left(\lambda_{r}\right)_{r \geq 1} \text { with } \sum\left|\lambda_{r}\right|^{p}=1,
$$


where $C>0$ depends only on $X$. Hence $\left(\sum_{r \geq 1}\left\|x_{r}\right\|^{p^{\prime}}\right)^{1 / p^{\prime}} \leq C\|f\|_{1}$. Thus, by a result of Pisier [Pi1] (cf. also [P]), $X$ has cotype $p^{\prime}$.

Remark. The proof of Theorem 2.4 actually shows that if $X$ has Fourier type $p$ then

(2.9) there exists $C>0$ such that for every $f \in H_{X}^{1}$, at and every $m \in F M_{p}$ $\sum_{j>0}\left\|\hat{f}(j) m_{j}\right\| \leq C\|f\|_{1, \text { at }}$.

We show next that (2.9) implies a slightly stronger property than cotype $p^{\prime}$ of $X$; namely,

Proposition 2.5. If $X$ satisfies (2.9) then $X^{*}$ has type $p$.

Proof. Similarly, as in the proof of Proposition 2.4, we show that (2.9) implies

$$
\left(\sum_{r=1}^{\infty}\left\|\hat{f}\left(2^{r}\right)\right\|^{p^{\prime}}\right)^{1 / p^{\prime}} \leq C\|f\|_{1, \text { at }} \text { for } f \in H^{1, \text { at }} .
$$

Now pick an eventually zero sequence $\left(x_{r}^{*}\right)_{r \geq 1}$ in $X^{*}$ and put $f^{*}=\sum_{r=1}^{\infty} x_{r} e^{i 2^{2} t}$. Clearly $\left\|f^{*}\right\|_{1} \leq\left\|f^{*}\right\|_{\text {BMO }}$. By Theorem 1.2, there is a numerical constant $C_{1}$ (independent of $f^{*}$ ) such that $\left\|f^{*}\right\|_{\mathrm{BMO}} \leq C_{1}\left\|f^{*}\right\|_{\left(H_{X}^{1, a t}\right)^{*}}$. Thus applying (2.10) and the Hölder inequality we get

$$
\begin{aligned}
& \left\|f^{*}\right\|_{\left(H_{X}^{1, \text { at })^{*}}\right.}=\sup \left\{\left|\left\langle f, f^{*}\right\rangle\right|: f \in H_{X}^{1, \text { at }},\|f\|_{1, \text { at }} \leq 1\right\} \\
& =\sup \left\{\left|\sum_{r \geq 1} x_{r}^{*}\left(\hat{f}\left(2^{r}\right)\right)\right|:\|f\|_{1, \text { at }} \leq 1\right\} \\
& \leq \sup \left\{\left(\sum_{r \geq 1}\left\|x_{r}^{*}\right\|^{p}\right)^{1 / p^{\prime}}\left(\sum_{r \geq 1}\left\|\hat{f}\left(2^{r}\right)\right\|^{p}\right)^{1 / p^{\prime}}:\|f\|_{1, \text { at }} \leq 1\right\} \\
& \leq C\left(\sum_{r \geq 1}\left\|x_{r}^{*}\right\|^{p}\right)^{1 / p^{\prime}} \text {. }
\end{aligned}
$$

Thus $\left\|f^{*}\right\|_{1} \leq C_{1} C\left(\sum_{r \geq 1}\left\|x_{r}^{*}\right\|^{p}\right)^{1 / p^{\prime}}$. Therefore, by Pisier's theorem $X^{*}$ has type $p$.

Applying Theorem 2.4 and Proposition 2.4 to $L^{q}$ and $S_{q}$ we get

Corollary 2.6. Let $1 \leq p<\infty$. Let $E_{p}$ denote either an infinite-dimensional $L^{p}(\nu)$ space or the trace class $S_{p}$. Then $m=\left(m_{j}\right) \in F M_{\min \left(2, p^{\prime}\right)}$ iff $m_{E_{p}}$ is $(1,1)$-bounded.

Proof. By Theorem 2.1, for every Banach space $X$ if $m_{X}$ is $(1,1)$-bounded then $m \in F M_{2}=F M$. Thus if $1 \leq p \leq 2$ then the desired conclusion follows from Corollary 2.2 for $E_{p}=L^{p}(\nu)$ and from Theorem 2.2 for $E_{p}=S_{p}$.

Let $p>2$. Then $\min \left(2, p^{\prime}\right)=p^{\prime}$. By a simple interpolation argument, $E_{p}$ is of Fourier type $p^{\prime}$ (cf. [Pee and MI]). Thus, by Theorem 2.4, if $m \in F M_{p^{\prime}}$ 
then $m_{E_{p}}$ is $(1,1)$-bounded. To prove the converse we modify an argument from [Sz-W]. First we have

Fact. If $m_{E_{p}}^{\prime}$ is $(\infty, 1)$-bounded, say with a bound $K$, then $\left(\sum_{k}\left|m_{k}^{\prime}\right|^{p^{\prime}}\right)^{1 / p^{\prime}} \leq$ $K$.

The fact is a direct consequence of

$1^{\circ} . l^{p}$ is finitely representable in $E_{p}$.

$2^{\circ}$. If $\left(c_{k}\right)_{k \geq 0}$ is an eventually zero scalar sequence, and if $\left(\delta_{k}\right)_{k \geq 0}$ is the unit vector basis of $l^{p}$, then

$$
\left\|\sum_{k \geq 0} c_{k} e^{i k t} \delta_{k}\right\|_{\infty}=\left(\sum_{k \geq 0}\left|c_{k}\right|^{p^{\prime}}\right)^{1 / p^{\prime}} .
$$

To complete the proof we shall show that if $m_{E_{p}}$ is $(1,1)$-bounded for some $m=\left(m_{k}\right)$ then for $s=1,2, \ldots \quad m_{E_{p}}^{\prime}$ and $m_{E_{p}}^{\prime \prime}$ are $(\infty, 1)$-bounded with a bound independent of $s$ where $m^{\prime}=\left(m_{k}^{\prime}\right)$ and $m^{\prime \prime}=\left(m_{k}^{\prime \prime}\right)$ are defined by $m_{0}^{\prime}=m_{0}^{\prime \prime}=0 ; \quad m_{k}^{\prime}=\sum_{0 \leq d<s / 2} m_{k s+d} ; \quad m_{k}^{\prime \prime}=\sum_{s / 2 \leq d<s} m_{k s+d}$ for $k=$ $1,2, \ldots$.

Let $f$ be an $E_{p}$-valued analytic trigonometric polynomial. Fix $s=1,2, \ldots$ and define $f_{s}$ by $f_{s}(t)=f(2 s t)$. Then $\hat{f}_{s}(n)=0$ for $n \neq 2 k s, \hat{f}_{s}(2 k s)=\hat{f}(k)$ for $k=0,1 \ldots$. Next put $g=f_{s} G$ where

$$
G(t)=\sum_{j^{\prime}=1}^{2 s-1}\left(1-\left|s-j^{\prime}\right| / s\right) e^{i t j^{\prime}} .
$$

Then for $n=2 k s+j$ with $k=0,1, \ldots, j=1,2, \ldots, 2 s-1$

$$
\hat{g}(n)=\sum_{j^{\prime}=1}^{2 s-1} \widehat{G}\left(j^{\prime}\right) \hat{f}_{s}\left(n-j^{\prime}\right)=\widehat{G}(j) \hat{f}(k) .
$$

Since $G$ is a shifted Fejér kernel, $\|G\|_{1}=1$. Thus $\|g\|_{1} \leq\left\|f_{s}\right\|_{\infty}\|G\|_{1}=\|f\|_{\infty}$. Hence, if $C$ is a $(1,1)$-bound for $m_{E_{p}}$, then

$$
\begin{aligned}
C\|f\|_{\infty} & \geq C\|g\|_{1} \geq \sum_{n \geq 0}\|\hat{g}(n)\|\left|m_{n}\right| \geq \sum_{k=0}^{\infty} \sum_{j=1}^{2 s-1}\left|m_{2 k s+j}\right| \widehat{G}(j)\|\hat{f}(k)\| \\
& \geq \sum_{k=0}^{\infty} \sum_{s \leq j<3 s / 2}\left|m_{2 k s+j}\right|\left\|\frac{\hat{f}(k)}{2}\right\|=\frac{1}{2} \sum_{k \geq 0}\left|m_{k}^{\prime}\right|\|\hat{f}(k)\|,
\end{aligned}
$$

because, for $s \leq j<3 s / 2, \widehat{G}(j) \geq \frac{1}{2}$. Thus $m_{E_{p}}^{\prime}$ is $(\infty, 1)$-bounded with a bound $2 C$. The verification of $(\infty, 1)$ boundedness of $M_{E_{p}}^{\prime \prime}$ is similar.

We end this section by discussing the relationship between the type of a Banach space and the vector-valued Hardy inequality. The main result is:

Theorem 2.5. If a Banach space $X$ has nontrivial type, then $X \in \mathscr{H} \mathscr{S}$. 
Theorem 2.5 is due to Jean Bourgain and is published here with his permission. Our proof is slightly different from Bourgain's original argument (which deals with the integral analogue of the Hardy inequality on the real line).

The proof is based on

Theorem 2.6. If $X$ has nontrivial type, then there are $q \in[2, \infty]$ and $C>0$, such that

$$
\left(\sum_{-\infty}^{+\infty}\|\hat{f}(n)\|^{q}\right)^{1 / q} \leq C\|f\|_{2} \quad \text { for } f \in L_{X}^{2} .
$$

Theorem 2.6 is a particular of [B3, Theorem 1].

An easy consequence of Theorem 2.6 is

Lemma 2.1. Let $X$ have nontrivial type, let $b$ be either a constant $X$-atom or an $X$-atom supported on $(-\pi, \pi)$. For $r$ real put $e_{r}(s)=e^{i r s}$ for $s \in R$. Then

$$
\left(\sum_{-\infty}^{+\infty}\left\|\left(b e_{r}\right)^{-}(n)\right\|^{q}\right)^{1 / q} \leq 2 C+1,
$$

where $C$ and $q$ are as in Theorem 2.6.

Proof. If $a$ is an $X$-atom supported on $(-\pi, \pi)$, then $\|a\|_{2} \leq\|a\|_{\infty} \leq 1$. Thus, by Theorem 2.6,

$$
\left(\sum_{-\infty}^{+\infty}\|\hat{a}(n)\|^{q}\right)^{1 / q} \leq C\|a\|_{2} \leq C .
$$

Write $b e_{r}=b^{(1)}+b^{(2)}$ where $b^{(2)}=(2 \pi)^{-1} \int_{-\pi}^{\pi}\left(b e_{r}\right)(s) d s \cdot 1$ and $b^{(1)}=b e_{r}-$ $b^{(2)}$. By the hypothesis on $b,\left\|b e_{r}\right\|_{\infty} \leq 1$. Hence $b^{(1)}$ is a multiple of an $X$-atom by a nonnegative number $\leq 2$. Thus

$$
\left(\sum_{-\infty}^{+\infty}\left\|\hat{b}^{(1)}(n)\right\|^{q}\right)^{1 / q} \leq 2 C .
$$

On the other hand, $b^{(2)}$ is a constant atom; therefore

$$
\left(\sum_{-\infty}^{+\infty}\left\|b^{(2)}(n)\right\|^{q}\right)^{1 / q}=\left\|\hat{b}^{(2)}(0)\right\| \leq 1 .
$$

Proof of Theorem 2.5. A standard reduction as in the proof of Theorem 2.4 shows that it is enough to prove

(2.11) There exists $K>0$ such that $\sum_{n \geq 0}\|\hat{a}(n)\|(n+1)^{-1} \leq K$ for every $X$-atom $a$ supported on $(-\pi \delta, \pi \delta)$ with $0<\delta \leq 1$.

Let $m$ be the integer satisfying $(m+1)^{-1}<\delta \leq m^{-1}$. Put

$$
S_{1}(a)=\sum_{n<m}\|\hat{a}(n)\|(n+1)^{-1} ; \quad S_{2}(a)=\sum_{n \geq m}\|\hat{a}(n)\|(n+1)^{-1} .
$$


By (1.1) and the choice of $m, S_{1}(a) \leq \sum_{n<m} 4^{-1} 2 \pi \delta n(n+1)^{-1} \leq \pi / 2$. To estimate $S_{2}(a)$ first observe that

$$
\left(\sum_{n \geq m}(n+1)^{-q^{\prime}}\right)^{1 / q^{\prime}} \leq\left(\int_{m}^{+\infty} x^{-q^{\prime}} d x\right)^{1 / q^{\prime}}=A(q) m^{-1 / q},
$$

where $A(q)=(q-1)^{(1-q) / q}$. Thus, by the Hölder inequality,

$$
S_{2}(a) \leq A(q) m^{-1 / q}\left(\sum_{n \geq m}\|\hat{a}(n)\|^{q}\right)^{1 / q} .
$$

Let $b$ be the periodic function on $R$ defined by $b(s)=\delta a(s / m)$ for $s \in$ $(-\pi, \pi)$, and let $b_{k}=b e_{-k / m}$ for $k=0,1, \ldots, m-1$. Note that $b$ is an $X$ atom supported on $(-\pi, \pi)$. Fix $n=m j+k$ for $j=1,2 \ldots$. Remembering that $a(t)=0$ for $\pi>|t|>\pi m^{-1}$ and substituting $s=m t$ we get

$$
\begin{aligned}
2 \pi \hat{a}(n) & =\int_{-\pi / m}^{\pi / m} a(t) e^{-i n t} d t=\int_{-\pi}^{\pi} m^{-1} a(s / m) e^{-i k s / m} e^{-i j s} d s \\
& =2 \pi(m \delta)^{-1} \hat{b}_{k}(j) .
\end{aligned}
$$

Thus $\|\hat{a}(n)\| \leq 2\left\|\hat{b}_{k}(j)\right\|$ because $m \delta \geq \frac{1}{2}$. Therefore, by Lemma 2.1,

$$
\sum_{n \geq m}\|\hat{a}(n)\|^{q} \leq 2^{q} \sum_{k=0}^{m-1} \sum_{j=1}^{\infty}\left\|\hat{b}_{k}(j)\right\|^{q} \leq 2^{q} \sum_{k=0}^{m-1} \sum_{-\infty}^{\infty}\left\|\hat{b}_{k}(j)\right\|^{q} \leq(4 C+2)^{q} m .
$$

Hence $S_{2}(a) \leq A(q) m^{-1 / q}(4 C+2) m^{1 / q}=A(q)(4 C+2)$. Thus we have established (2.11) with $K \leq A(q)(4 C+2)+\pi / 2$.

Theorem 2.5 yields yet another characterization of Banach spaces with a nontrivial type.

Proposition 2.6. For every Banach space $X$ the following are equivalent:

(+) $X$ has a nontrivial type,

$(++)$ there is a $K>0$ such that for every $f \in H_{X}^{1, \text { at }}$

$$
\sum_{n \geq 0}\|\hat{f}(n)\|(n+1)^{-1} \leq K\|f\|_{1, \text { at }} .
$$

Proof. $(+) \Rightarrow(++)$. This implication follows from (2.11).

$(++) \Rightarrow(+)$. First note that if a Banach space $Y$ fails $(++)$ then for every $K>0$ there is a $Y$-valued trigonometric polynomial, say $f$, such that $\|f\|_{1, \text { at }} \leq 1$ and $\sum_{n \geq 0}\|\hat{f}(n)\|(n+1)^{-1}>K$. Now a routine argument shows that if $Y$ fails $(++)$ and $Y$ is crudely finitely representable in $X$, then $X$ fails $(++)$. (Note only that if $E$ is a subspace of $X$, and $f$ is an $E$-valued trigonometric polynomial, then $\|f\|_{H_{E}^{1, \text { at }}} \geq\|f\|_{H_{X}^{1 . \text { at }}}$.) Furthermore, it is well known (cf. [M-P]) that if $p_{X}=1$ then $L^{1}$ is finitely represented in $X$. Thus 
to prove the implication "not $(+) " \Rightarrow$ "not $(++)$ " it is enough to show that $L^{1}$ fails $(++)$. To this end given $K>0$ pick a trigonometric polynomial $F$ so that $\|F\|_{L^{1}}=1, \int_{-\pi}^{\pi} F(t) d t=2 \pi \widehat{F}(0)=0$ and $\sum_{n \geq 1}|\hat{F}(n)|(n+1)^{-1}>K$. Next define an $L^{1}$-valued trigonometric polynomial $f$ by $f(s)=F_{s}$ where $F_{s}(t)=F(s+t)$. Clearly $\hat{f}(n)=F(n) e_{n}$ where $e_{n}(t)=e^{i n t}$. Thus $f(s)=$ $\sum_{n \neq 0} \widehat{F}(n) e_{n} e^{i n s}$. Hence $\int_{-\pi}^{\pi} f(s) d s=0$. Obviously $\|f(s)\|_{L^{1}}=\left\|F_{s}\right\|_{L^{1}}=$ $\|F\|_{L^{1}}=1$ for $s \in R$. Thus $f$ is an $L^{1}$-atom supported by $(-\pi, \pi)$. Hence $\|f\|_{1, \text { at }} \leq 1$.

On the other hand

$$
\sum_{n \geq 0}\|\hat{f}(n)\|_{L^{1}}(n+1)^{-1}=\sum_{n \geq 0}\|\widehat{F}(n)\|_{L^{1}}(n+1)^{-1}>K .
$$

Remark. Theorem 2.5 obviously improves Corollary 2.5 because a Banach space with nontrivial Fourier type has nontrivial type (well known and easy). After the preliminary version of this paper had been written, J. Bourgain [B4] proved in August 1987 (solving a problem which has been open for a while, cf. [Mi]) the converse:

Every Banach space with type $>1$ has Fourier type $>1$. This gives via Corollary 2.5 yet another proof of Theorem 2.5 .

\section{Paley spaces}

Recall (cf. [Du, p. 103]) that $m=\left(m_{j}\right)_{j \geq 0}$ is $\left(H^{1}-l^{2}\right)$-multiplier iff

$$
\sigma(m)=\left(\left|m_{0}\right|^{2}+\sup _{n \geq 1} n^{-2} \sum_{j=1}^{n} j^{2}\left|m_{j}\right|^{2}\right)^{1 / 2}<+\infty .
$$

Denote by $P M$ the Banach space (under the norm $\sigma(\cdot)$ ) of all sequences $m$ satisfying (3.1).

Definition 3.1. A Banach space $X$ is called a Paley space provided for every $\left(H^{1}-l^{2}\right)$-multiplier $m$ the induced operator $m_{X}$ is $(1,2)$-bounded.

Recall that given $q>1$ a sequence $\left(n_{k}\right)_{k \geq 1}$ of positive integers is called $q$-lacunary for some $q>1$ provided

$$
\inf _{k} n_{k}^{-1} n_{k+1} \geq q
$$

A lacunary sequence is a $q$-lacunary sequence for some $q>1$.

Our first result collects several equivalent conditions for a Banach space of being a Paley space.

Proposition 3.1. Let $X$ be a Banach space. Then the following are equivalent:

(i) $X$ is a Paley space, 
(ii) there is $C>0$ such that for every $f \in H_{X}^{1}$ and every $m \in P M$

$$
\left(\sum_{j>0}\left\|m_{j} \hat{f}(j)\right\|^{2}\right)^{1 / 2} \leq C \sigma(m)\|f\|_{1},
$$

(iii) for every lacunary sequence $\left(n_{k}\right)_{k \geq 1}$ there is a constant $C_{\left(n_{k}\right)}>0$ such that for every $X$-valued analytic trigonometric polynomial $f$

$$
\sum_{k \geq 1}\left\|\hat{f}\left(n_{k}\right)\right\|^{2} \leq\left[C_{\left(n_{k}\right)}\right]^{2}\|f\|_{1}^{2}
$$

(iv) for some $q>1$ there is a constant $C_{q}>0$ such that for every q-lacunary sequence $\left(n_{k}\right)$ and every $X$-valued analytic trigonometric polynomial $f$

$$
\sum_{k \geq 1}\left\|\hat{f}\left(n_{k}\right)\right\|^{2} \leq C_{q}^{2}\|f\|_{1}^{2}
$$

(v) for some $q>1$ there is a constant $C_{q}>0$ such that for every eventually zero sequence $\left(x_{k}^{*}\right)_{k \geq 1}$ in $X^{*}$ and every q-lacunary sequence $\left(n_{k}\right)$ there is an $X^{*}$-valued trigonometric polynomial $g^{*}$ such that

$$
\begin{array}{r}
\|g\|_{\infty}^{2} \leq C_{q}^{2} \sum_{k \geq 1}\left\|x_{k}^{*}\right\|^{2}, \quad \hat{g}\left(n_{k}\right)=x_{k}^{*} \quad \text { for } k=1,2, \ldots, \\
\hat{g}(j)=0 \quad \text { for } j \geq 0, \quad j \neq n_{1}, n_{2}, \ldots
\end{array}
$$

Proof. (i) $\Rightarrow$ (ii). Use a Baire category argument.

(ii) $\Rightarrow$ (iii). Observe that every lacunary sequence belongs to $P M$.

(iii) $\Rightarrow$ (iv). If (iv) were false then one could define inductively a sequence $\left(f_{r}\right)$ of $X$-valued trigonometric polynomials (each $f_{r}$ of degree $N_{r}$ respectively) and a sequence $\left(\left(n_{k}^{(r)}\right)_{k \geq 1}\right)_{r \geq 1}$ of $q$-lacunary sequences such that $N_{1} \geq 1$, and for $r=1,2, \ldots$

$$
\left\|f_{r}\right\|_{1}=1, \quad \sum_{k}\left\|\hat{f}_{r+1}\left(n_{k}^{(r+1)}\right)\right\| \geq 2 N_{r} ; \quad N_{r+1} \geq q N_{r} .
$$

Let $\left(n_{k}\right)_{k \geq 1}$ be the enumeration in the increasing order of the set

$$
\bigcup_{r \geq 2} \bigcup_{N_{r-1}<l<N_{r}}\left\{n_{l}^{(r)}\right\} \text {. }
$$

Then $\left(n_{k}\right)_{k \geq 1}$ is a lacunary sequence, and for $r=2,3, \ldots$

$$
\begin{aligned}
\sum_{k \geq 1}\left\|\hat{f}_{r}\left(n_{k}\right)\right\|^{2} & \geq \sum_{l=1}^{N_{r}}\left\|\hat{f}_{r}\left(n_{l}^{(r)}\right)\right\|^{2}-\sum_{l=1}^{N_{r-1}}\left\|\hat{f}_{r}\left(n_{l}^{(r)}\right)\right\|^{2} \\
& \geq 2 N_{r-1}-N_{r-1} \geq N_{r-1},
\end{aligned}
$$

because $\left\|f_{r}\right\|_{1}=1$ yields $\left\|\hat{f}_{r}(j)\right\| \leq 1$ for all $j$. Thus the lacunary sequence $\left(n_{k}\right)_{k \geq 1}$ would not satisfy (iii), a contradiction. 
(iv) $\Rightarrow(v)$. The proof is similar to that of the implication (ii) $\Rightarrow$ (iii) of Proposition 2.1.

$(\mathrm{v}) \Rightarrow(\mathrm{i})$. Let $q$ and $C_{q}$ be as in (v). Let $m \in P M$. Fix $q_{0}>q$. Then

$$
\sum_{q_{0}^{k-1}}^{q_{0}^{k}-1}\left|m_{j}\right|^{2} \leq q_{0}^{2} \sum_{1}^{q_{0}^{k}}\left|m_{j}\right|^{2} j^{2} q_{0}^{-2 k} \leq \sigma(m)^{2} q_{0}^{2} \text {. }
$$

Fix $f \in H_{X}^{1}$. For $k=1,2, \ldots$ pick $n_{k}$ with $q_{0}^{k-1} \leq n_{k}<q_{0}^{k}$ so that $\left\|\hat{f}\left(n_{k}\right)\right\|^{2}=\max \left\{\|\hat{f}(j)\|^{2}: q_{0}^{k-1} \leq j<q_{0}^{k}\right\}$. Obviously $\left(n_{2 k-1}\right)$ and $\left(n_{2 k}\right)$ are $q$-lacunary sequences and

$$
\sum_{q_{0}^{k-1}}^{q_{0}^{k}-1}\|\hat{f}(j)\|^{2}\left|m_{j}\right|^{2} \leq[\sigma(m)]^{2} q_{0}^{2}\left\|\hat{f}\left(n_{k}\right)\right\|^{2} \quad \text { for } k=1,2, \ldots
$$

Next fix a positive integer $N$ and choose for $k=1,2, \ldots$ functionals $x_{k}^{*}$ and $y_{k}^{*}$ in $X^{*}$ so that

$$
\begin{aligned}
\sum_{k=1}^{N}\left\|x_{k}^{*}\right\|^{2} & =\sum_{k=1}^{N}\left\|y_{k}^{*}\right\|^{2}=1, \\
\sum_{k=1}^{N} x_{k}^{*}\left(\hat{f}\left(n_{2 k-1}\right)\right) & =\left(\sum_{k=1}^{N}\left\|\hat{f}\left(n_{2 k-1}\right)\right\|^{2}\right)^{1 / 2} ; \\
\sum_{k=1}^{N} y_{k}^{*}\left(\hat{f}\left(n_{2 k}\right)\right) & =\left(\sum_{k=1}^{N}\left\|\hat{f}\left(n_{2 k}\right)\right\|^{2}\right)^{1 / 2} .
\end{aligned}
$$

It follows from $(\mathrm{v})$ that there are $X^{*}$-valued trigonometric polynomials $g_{1}^{*}$ and $g_{2}^{*}$ such that $\left\|g_{1}^{*}\right\|_{\infty}+\left\|g_{2}^{*}\right\|_{\infty} \leq 2 C_{q}, \quad \hat{g}_{1}^{*}\left(n_{2 k-1}\right)=x_{k}^{*}, \quad \hat{g}_{2}^{*}\left(n_{2 k}\right)=y_{k}^{*}$ for $k=1,2, \ldots, N, \hat{g}_{1}^{*}(j)=0$ for $j \geq 0$ and $j \neq n_{1}, n_{3}, \ldots ; \hat{g}_{2}^{*}(j)=0$ for $j \geq 0$ and $j \neq n_{2}, n_{4}, \ldots$. Clearly

$$
\begin{aligned}
\left\langle f, g_{1}^{*}+g_{2}^{*}\right\rangle & =\left(\sum_{k=1}^{N}\left\|\hat{f}\left(n_{2 k-1}\right)\right\|^{2}\right)^{1 / 2}+\left(\sum_{k=1}^{N}\left\|\hat{f}\left(n_{2 k}\right)\right\|^{2}\right)^{1 / 2} \\
& \leq\|f\|_{1}\left\|g_{1}^{*}+g_{2}^{*}\right\|_{\infty} \leq 2 C_{q}\|f\|_{1} .
\end{aligned}
$$

Hence

$$
\begin{aligned}
& \sum_{j=0}^{q_{0}^{2 N}-1}\|\hat{f}(j)\|^{2}\left|m_{j}\right|^{2}=\|\hat{f}(0)\|^{2}\left|m_{0}\right|^{2}+\sum_{k=1}^{2 N} \sum_{q_{0}^{k-1}}^{q_{0}^{k}-1}\|\hat{f}(j)\|^{2}\left|m_{j}\right|^{2} \\
& \leq \hat{f}(0) \|^{2}\left|m_{0}\right|^{2}+[\sigma(m)]^{2} q_{0}^{2}\left(\left(\sum_{k=1}^{N}\left\|\hat{f}\left(n_{2 k-1}\right)\right\|^{2}\right)^{1 / 2}+\left(\sum_{k=1}^{N}\left\|\hat{f}\left(n_{2 k}\right)\right\|^{2}\right)^{1 / 2}\right)^{2} \\
& \leq[\sigma(m)]^{2}\left(1+4 C_{q}^{2} q_{o}^{2}\right)\|f\|_{1}^{2} .
\end{aligned}
$$

Thus $m$ is $(1,2)$-bounded. 
R. E. A. C. Paley proved in $[\mathrm{Pa}]$ that characteristic functions of lacunary sequences are $\left(H^{1}-l^{2}\right)$-multipliers. This together with the equivalence (i) $\Rightarrow(\mathrm{v})$ motivate the name "Paley spaces".

We begin the discussion of Paley spaces with the obvious

Corollary 3.1. Every space which is crudely finitely representable in a Paley space is itself a Paley space.

Next we have (compare with Proposition 2.4):

Proposition 3.2. If $X$ is a Paley space then $X$ is cotype 2 .

Proof. For an arbitrary eventually zero sequence $\left(x_{k}\right)_{k \geq 1}$ in $X$ put $f=$ $\sum_{k \geq 1} x_{k} e^{i 2^{k} t}$. Since $X$ is a Paley space, there is an absolute constant $C_{2}$ (independent of the sequence $\left.\left(x_{k}\right)_{k \geq 1}\right)$ such that

$$
\sum\left\|\hat{f}\left(2^{k}\right)\right\|^{2}=\sum\left\|x_{k}\right\|^{2} \leq C_{2}^{2}\|f\|_{1}^{2} .
$$

Thus, by a result of Pisier (cf. [Pi1] and [P]), $X$ has cotype 2 .

Remarks. $1^{\circ}$. The space $L^{1} / \bar{H}_{0}^{1}$ is of cotype 2 (cf. [B2]), but it is not a Paley space. Indeed define $m=\left(m_{j}\right)$ by $m_{2^{k}}=k^{-1}$ for $k=1,2, \ldots, m_{j}=0$ for $j \geq 0, j \neq 2,4,8, \ldots$ If $L^{1} / \bar{H}_{0}^{1}$ were a Paley space, then the operator $m_{L^{1} / \bar{H}_{0}}$ induced by $m$ would be $(1,1)$-bounded. This would contradict Proposition 1.1.

$2^{\circ}$. For ever $p$ with $1<p<2$ there are spaces in the class $\mathscr{F}_{p}$ which are not of cotype 2 (for instance $L^{p^{\prime}}$ or $S_{p^{\prime}}$ ). Thus for $p<2$ the class $\mathscr{F}_{p}$ (as well as $\mathscr{H} \mathscr{F}$ ) is not contained in the class of Paley spaces. However, we have

Proposition 3.3. Every space of $\left(H^{1}-l^{1}\right)$-Fourier type is a Paley space.

Proof. Let $m=\left(m_{j}\right)$ be an $\left(H^{1}-l^{1}\right)$-multiplier. Then for every sequence $\left(\lambda_{j}\right)_{j \geq 0} \in l^{2}$ the sequence $\left(\lambda_{j} m_{j}\right)_{j \geq 0}$ is a $\left(H^{1}-l^{1}\right)$-multiplier. Hence, if $X$ is of $\left(H^{1}-l^{1}\right)$-Fourier type then for every $f \in H_{X}^{1}, \sum_{j>0}\left|\lambda_{j} m_{j}\right|\|\hat{f}(j)\|<\infty$ for every $\left(\lambda_{j}\right) \in l^{2}$. Hence, by the Landau theorem, $\sum_{j \geq 0}\left|m_{j}\right|^{2}\|\hat{f}(j)\|^{2}<\infty$. Now a standard Baire category argument yields that $m_{x}$ is $(1,2)$-bounded.

We shall show next that a slightly stronger assumption than "cotype 2 " already implies that a space is Paley.

Theorem 3.1. If $X^{*}$ is of type 2, then $X$ is a Paley space.

Proof. In view of Theorem 1.1 it is enough to prove

Proposition 3.4. If $X^{*}$ has type 2 , then for every $q>1$ there is a constant $C_{q}$ such that

(3.4) for every q-lacunary sequence $\left(n_{k}\right)_{k \geq 1}$ and every $f \in H_{X}^{1}$, at

$$
\sum_{k \geq 1}\left\|\hat{f}\left(n_{k}\right)\right\|_{X}^{2} \leq C_{q}^{2}\|f\|_{1, \text { at }}^{2} .
$$


Proof. It is enough to show that there is a constant $C_{q}$ such that for every $q$-lacunary sequence $\left(n_{k}\right)$ and for every $X$-atom $a$

$$
\sum=\sum_{k \geq 1} \mid \hat{a}\left(n_{k}\right) \|^{2} \leq C_{q}^{2} .
$$

This is trivial for constant atoms. Otherwise it is enough to consider atoms supported on the interval $[-\pi \delta, \pi \delta]$ for some $\delta$ with $0<\delta \leq 1$ (because a translate of an atom is again an atom). Let $k_{0}$ be the positive integer satisfying $n_{k_{0}} \leq \delta^{-1}<n_{k_{0}}+1$. Write $\Sigma=\Sigma_{1}+\Sigma_{2}$ where

$$
\Sigma_{1}=\sum_{k=1}^{k_{0}}\left\|\hat{a}\left(n_{k}\right)\right\|^{2}, \quad \Sigma_{2}=\sum_{k>k_{0}}\left\|\hat{a}\left(n_{k}\right)\right\|^{2} .
$$

To estimate $\Sigma_{1}$ observe that (3.2) implies

$$
n_{k} n_{k_{0}}^{-1} \leq \prod_{j=k}^{k_{0}-1} n_{j} n_{j+1}^{-1} \leq q^{k-k_{0}} \quad \text { for } 1 \leq k \leq k_{0} .
$$

Now using the estimate $\|\hat{a}(j)\| \leq \pi \delta|j| / 8$ for $j=0, \pm 1, \pm 2$ which follows from (1.1) we obtain

$$
\Sigma_{1} \leq \frac{\pi^{2}}{64} \sum_{k=1}^{k_{0}}\left(n_{k} \delta\right)^{2} \leq \frac{\pi^{2}}{64} \sum_{k=1}^{k_{0}}\left(n_{k} n_{k_{0}}^{-1}\right)^{2} \leq \frac{\pi^{2}}{64} \cdot \frac{q^{2}}{q^{2}-1} .
$$

To estimate $\Sigma_{2}$, for every integer $N>k_{0}$ pick a sequence $\left(x_{k}^{*}\right)_{k_{0}<k \leq N}$ in $X^{*}$ so that

$$
\begin{gathered}
\sum_{k=k_{0}+1}^{N}\left\|x_{k}^{*}\right\|^{2}=1, \quad x_{k}^{*}\left(\hat{a}\left(n_{k}\right)\right) \geq 0 \text { for } k=1,2, \ldots, \\
\left(\sum_{k=k_{0}+1}^{N}\left\|\hat{a}\left(n_{k}\right)\right\|^{2}\right)^{1 / 2}=\sum_{k=k_{0}+1}^{N} x_{k}^{*}\left(\hat{a}\left(n_{k}\right)\right) .
\end{gathered}
$$

Put

$$
f_{N}^{*}(t)=\sum_{k=k_{0}+1}^{N} x_{k}^{*} e^{i n_{k} t}
$$

Remembering that $a$ is supported on $[-\pi \delta, \pi \delta]$ and therefore $\|a\|_{\infty} \leq \delta^{-1}$ we have

$$
\begin{aligned}
\sum_{k=k_{0}+1}^{N} x_{k}^{*}\left(\hat{a}\left(n_{k}\right)\right) & =\left\langle a, f_{N}^{*}\right\rangle=(2 \pi)^{-1} \int_{-\pi \delta}^{\pi \delta}\left[f_{N}^{*}(t)\right](a(-t)) d t \\
& \leq(2 \pi)^{-1}\|a\|_{\infty} \int_{-\pi \delta}^{\pi \delta}\left\|f_{N}^{*}(t)\right\|_{X^{*}} d t \\
& \leq(2 \pi \delta)^{-1} \int_{-\pi \delta}^{\pi \delta}\left\|f_{N}^{*}(t)\right\|_{X^{*}} d t
\end{aligned}
$$


Substituting $t=s \delta$ we get

$$
(2 \pi \delta)^{-1} \int_{-\pi \delta}^{\pi \delta}\left\|f_{N}^{*}(t)\right\|_{X^{*}} d t=(2 \pi)^{-1} \int_{-\pi \delta}^{\pi \delta}\left\|f_{N}^{*}(s \delta)\right\|_{X^{*}} d t
$$

Observe that

$$
f_{N}^{*}(s \delta)=\sum_{k=k_{0}+1}^{N} x_{k}^{*} e^{i \lambda_{k} s}
$$

where $\lambda_{k}=n_{k} \delta$ for $k=k_{0}+1, k_{0}+2, \ldots$. Clearly $\left(\lambda_{k}\right)_{k>k_{0}}$ is a sequence of real numbers such that $\lambda_{k_{0}+1} \geq n_{k_{0}+1}\left(n_{k_{0}}+1\right) \geq 1$ and $\inf _{k>k_{0}} \lambda_{k+1} \lambda_{k}^{-1} \geq q>1$. Thus, by [P, Theorem 3], there is a constant $C(q)$ (depending only on $q$ ) such that

$$
(2 \pi)^{-1} \int_{-\pi}^{\pi}\left\|f_{N}^{*}(s \delta)\right\|_{X^{*}} d s \leq C(q) \int_{0}^{1}\left\|\sum_{j=1}^{N-k_{0}+1} r_{j}(\omega) x_{j+k_{0}}^{*}\right\| d \omega .
$$

Hence if $T_{2}$ is a type 2 constant of $X^{*}$ then

$$
(2 \pi)^{-1} \int_{-\pi}^{\pi}\left\|f_{N}^{*}(s \delta)\right\|_{X^{*}} d s \leq C(q) T_{2}\left(\sum_{k_{0}+1}^{N}\left\|x_{k}^{*}\right\|^{2}\right)^{1 / 2} \leq C(q) T_{2} .
$$

Thus

$$
\left(\sum_{k_{0}+1}^{N}\left\|\hat{a}\left(n_{k}\right)\right\|^{2}\right)^{1 / 2} \leq C(q) T_{2} .
$$

Consequently $\Sigma_{2} \leq C(q)^{2} T_{2}^{2}$. This completes the proof of (3.5) with

$$
C_{q}^{2}=\frac{\pi^{2}}{64} \frac{q^{2}}{q^{2}-1}+C(q)^{2} T_{2}^{2}
$$

Observe that in fact (3.4) characterizes Banach spaces whose first duals are of type 2. Precisely we have

Proposition 3.5. If $X$ is a Banach space such that for some $q>1$ there is a constant $C_{q}$ such that (3.4) holds, then $X^{*}$ is of type 2.

The proof of Proposition 3.5 is similar to that of Proposition 2.5.

Next we discuss the case of complex Banach lattices.

Let $X$ be an arbitrary Banach space and let $\left(E,\|\cdot\|_{E}\right)$ be a Banach lattice of absolutely integrable scalar functions on a probability space $(\Omega, \mu)$ (cf. e.g. [LT, Chapter I]). By $E_{X}$ we denote the space of $X$-valued Bochner $\mu$-integrable functions $f$ on $\Omega$ such that the function $\omega \rightarrow\|f(\omega)\|_{X}$ belongs to $E$ equipped with the norm

$$
\|f\|_{E_{X}}=\|\| f(\cdot)\left\|_{X}\right\|_{E} \cdot
$$

Recall that if $\left(E,\|\cdot\|_{E}\right) \subset L^{1}(\mu)$ is as above, then the lattice norm $\|\cdot\|_{E}$ is said to be two concave provided there is a constant $A$ such that for every finite 
sequence $\left(f_{k}\right)$ in $E$

$$
\left(\sum_{k}\left\|f_{k}\right\|_{E}^{2}\right)^{1 / 2} \leq A\left\|\left(\sum_{k}\left|f_{k}\right|^{2}\right)^{1 / 2}\right\|_{E}
$$

With these concepts we have

Proposition 3.6. If $X$ is a Paley space and $\left(E,\|\cdot\|_{E}\right)$ is a two concave function lattice then $E_{X}$ is a Paley space.

Proof. Let $\left(n_{k}\right)$ be a fixed lacunary sequence. Let $F$ be an $E_{X}$-valued analytic trigonometric polynomial. Then $F(\omega)$ is an $X$-valued analytic trigonometric polynomial and $F(\omega) \wedge(j)=\widehat{F}(j)(\omega) \quad \mu$-almost everywhere for all $j$. Since $X$ is a Paley space, there is a constant $C=C\left(X,\left(n_{k}\right)\right)>0$ such that $\mu$-almost everywhere

$$
\left(\sum_{k}\left\|\widehat{F}\left(n_{k}\right)(\omega)\right\|_{X}^{2}\right)^{1 / 2} \leq C(2 \pi)^{-1} \int_{-\pi}^{\pi}\|(F(\omega))(t)\|_{X} d t
$$

Since $\|\cdot\|_{E}$ is a lattice norm, the latter inequality implies

$$
\left\|\left(\sum_{k}\left\|\widehat{F}\left(n_{k}\right)(\cdot)\right\|_{X}^{2}\right)^{1 / 2}\right\|_{E} \leq C(2 \pi)^{-1}\left\|\int_{-\pi}^{\pi}\right\| F(\cdot)(t)\left\|_{X} d t\right\|_{E} .
$$

Since the norm of the integral is dominated by the integral of the norm, we get

$$
C(2 \pi)^{-1}\left\|\int_{-\pi}^{\pi}\right\| F(\cdot)(t)\left\|_{X} d t\right\|_{E} \leq C(2 \pi)^{-1} \int_{-\pi}^{\pi}\|\| F(\cdot)(t)\|\|_{E} d t=C\|F\|_{1} .
$$

On the other hand, the two concavity of the norm $\|\cdot\|_{E}$ implies

$$
\begin{aligned}
\left(\sum_{k}\left\|\widehat{F}\left(n_{k}\right)\right\|_{E_{X}}^{2}\right)^{1 / 2} & =\left(\sum\|\| \widehat{F}\left(n_{k}\right)(\cdot)\left\|_{X}\right\|_{E}^{2}\right)^{1 / 2} \\
& \leq A\left\|\left(\sum_{k}\left\|\widehat{F}\left(n_{k}\right)(\cdot)\right\|_{X}^{2}\right)^{1 / 2}\right\|_{E} .
\end{aligned}
$$

Thus

$$
\left(\sum_{k}\left\|\widehat{F}\left(n_{k}\right)\right\|_{E_{X}}^{2}\right)^{1 / 2} \leq C A\|F\|_{1}
$$

Corollary 3.2. Let $1 \leq p \leq 2$. Then for every measure space ( $\nu \Xi)$ and every Paley space $X$ the space $L_{X}^{p}(\nu)$ is a Paley space.

It is well known that $L_{X}^{p}(\nu)$ is finitely represented in $L_{X}^{p}$. For $1 \leq p \leq 2$, $L^{p}$ is a complex two concave Banach lattice on a probability space. Thus the desired conclusion follows from Corollary 3.1 and Proposition 3.6. 
Another simple consequence of Proposition 3.6 is

Theorem 3.2. A complex Banach lattice is a Paley space iff it is of cotype 2.

Proof. Let $E$ be a Banach lattice of cotype 2. Since a Banach space is Paley iff every separable subspace is Paley, we can assume without loss of generality that $E$ is separable. Thus we can also assume that $E$ being of cotype 2 is a lattice of integrable functions on a probability space $(\Omega, \mu)$ and the lattice norm of $E$ is two concave (cf. e.g. [L-T, Chapter I]). Now we apply Proposition 3.6 for $X=\mathbb{C}$. The converse implication follows from Proposition 3.2.

Finally we shall show

Thoerem 3.3. The dual of a $C^{*}$-algebra is a Paley space.

The proof is based on two lemmas. The first exploits the $C^{*}$-algebra structure via the von Neumann inequality (cf. [H, No. 229]).

Lemma 3.1. If $X$ is a $C^{*}$-algebra, then

$$
\left\|2^{-1} \alpha s+\left(1-s^{2}\right) a-2 s \bar{\alpha} a^{2}\right\| \leq 1
$$

for $a \in X$ with $\|a\| \leq 1, \alpha \in \mathbb{C}$ with $|\alpha|=1$, and $0 \leq s \leq 1$.

Proof. Let $P(\beta)=2^{-1} \alpha s+\left(1-s^{2}\right) \beta-2^{-1} s \bar{\alpha} \beta^{2}$. Then by the von Neumann inequality

$$
\|P(a)\| \leq \sup _{|\beta|=1}|P(\beta)| \quad \text { for } a \in X \text { with }\|a\| \leq 1
$$

Let $\beta=e^{i \theta}, \alpha=e^{i \theta_{0}}$. Then $2^{-1}(\alpha \bar{\beta}-\beta \bar{\alpha})=i \sin \left(\theta_{0}-\theta\right)$. Thus

$$
\sup _{|\beta|=1}|P(\beta)|=\sup _{0 \leq \theta<2 \pi} \mid \text { is } \sin \left(\theta_{0}-\theta\right)+1-s^{2} \mid \leq 1 \text {. }
$$

Lemma 3.1 allows one to adopt the one-sided Riesz product construction (cf. [Sm]) for functions with values in a $C^{*}$-algebra.

Lemma 3.2. Let $\left(u_{k}\right)_{1 \leq k \leq N}$ be a finite sequence of unitary elements of a $C^{*}$ algebra $X$ and let $\left(s_{k}\right)_{1 \leq k \leq N}$ be a sequence of nonnegative numbers such that $\sum_{k=1}^{N} s_{k}^{2}=4^{-1}$.

Then for every $q>2$ and every q-lacunary sequence $\left(n_{k}\right)_{k \geq 1}$ there is an $X$-valued trigonometric polynomial $g$ such that

$$
\begin{aligned}
\hat{g}\left(n_{N}\right)=2^{-1} s_{N} u_{N} ; \quad \hat{g}\left(n_{k}\right)=2^{-1} s_{k} \prod_{j=k+1}^{N}\left(1-s_{j}^{2}\right) u_{k} \\
\quad \text { for } k=1,2, \ldots, N-1,
\end{aligned}
$$

$$
\hat{g}(n)=0 \text { for } n>0 \text { and } n \neq n_{1}, n_{2}, \ldots
$$


Proof. Define inductively a sequence $\left(g_{k}\right)_{1 \leq k \leq N}$ of $X$-valued trigonometric polynomials by

$$
\begin{gathered}
g_{1}=2^{-1} s_{1} u_{1} e^{i n_{1} t}, \\
g_{k}=2^{-1} s_{k} u_{k} e^{i n_{k} t}+\left(1-s_{k}^{2}\right) g_{k-1}-2^{-1} s_{k} g_{k-1} u_{k}^{-1} g_{k-1} e^{-i n_{k} t} \\
\quad \text { for } k=2,3, \ldots, N .
\end{gathered}
$$

Then $g_{m}$ satisfies (3.6), (3.7), (3.8) with $N$ replaced by $m$ and $g$ replaced by $g_{m}$. Indeed assume that, for some $m>1, g_{m-1}$ satisfies (3.6)-(3.8). Fix $t \in(-\pi, \pi)$, put $s_{m}=s, \alpha=e^{i n_{m} t}, a=g_{m-1}(t) u_{m}^{-1}$. The hypothesis $\left\|g_{m-1}\right\|_{\infty} \leq 1$ implies $\|a\|=\left\|g_{m-1}(t)\right\| \leq 1$. Thus, by Lemma 3.1, $\left\|g_{m}(t) u_{m}^{-1}\right\| \leq 1$. Thus $\left\|g_{m}(t)\right\| \leq 1$ because $u_{m}$ is a unitary element. Hence $g_{m}$ satisfies (3.6). Next observe that the assumption that $g_{m-1}$ satisfies (3.7) and (3.8) implies $\left(g_{m-1} u_{m} g_{m-1}\right)^{\wedge}(n)=0$ for $n \geq 2 n_{m-1}$. Thus the condition $n_{m} \cdot n_{m-1}^{-1} \geq q>2$ implies that all nonnegative Fourier coefficients of the polynomial $2^{-1} s_{m} g_{m-1} u_{m}^{-1} g_{m-1} e^{-i n_{m} t}$ vanish. Now it is evident that if $g_{m-1}$ satisfies (3.7) and (3.8), then so does $g_{m}$.

We put $g=g_{N}$.

Proof of Theorem 3.3. Let $X$ be a $C^{*}$-algebra. Since $X^{*}$ embeds isometrically into $X^{* * *}$, and $X^{* *}$ is a unital $C^{*}$-algebra, we may assume that $X$ is a unital $C^{*}$-algebra. Let $f$ be an $X^{*}$-valued analytic trigonometric polynomial and let $\left(n_{k}\right)$ be a $q$-lacunary sequence for some $q>2$. Put $S=\sum_{k}\left\|\hat{f}\left(n_{k}\right)\right\|^{2}$. If $S=0$ there is nothing to prove. If $S \neq 0$, fix $N$ so large that $\hat{f}\left(n_{k}\right)=0$ for $k>N$. Put $x_{k}^{*}=\hat{f}\left(n_{k}\right) \cdot(2 \sqrt{S})^{-1}, s_{k}=\left\|x_{k}^{*}\right\|(k=1,2, \ldots, N)$. Then $\sum_{k=1}^{N} s_{k}^{2}=4^{-1}$. By the Russo-Dye theorem [Bo-Dn, p. 210], there exists a sequence $\left(u_{k}\right)_{1 \leq k \leq N}$ of unitary elements of $X$ such that

$$
s_{k} \geq x_{k}^{*}\left(u_{k}\right) \geq 2^{-1} s_{k} \text { for } k=1,2, \ldots, N .
$$

Let $g$ be an $X$-valued trigonometric polynomial satisfying (3.6)-(3.8) for the sequences $\left(n_{k}\right),\left(s_{k}\right)$ and $\left(u_{k}\right)$. Then

$$
\left\langle g,(2 \sqrt{S})^{-1} f\right\rangle=\sum_{k} s_{k} \prod_{j=k+1}^{N}\left(1-s_{k}^{2}\right) x_{k}^{*}\left(u_{k}\right) \geq 2^{-1} \sum_{k} s_{k}^{2} \prod_{j=k+1}^{N}\left(1-s_{k}^{2}\right) .
$$

Next observe that the condition $\sum_{k=1}^{N} s_{k}^{2}=4^{-1}$ implies that

$$
\inf _{k} \prod_{j=k+1}^{N}\left(1-s_{k}^{2}\right) \geq c>0
$$

where $c$ is a numerical constant independent both of $N$ and of the sequence $\left(s_{k}\right)$. Thus

$$
\left\langle g,(2 \sqrt{S})^{-1} f\right\rangle \geq 2^{-1} c \Sigma s_{k}^{2}=8^{-1} c .
$$


On the other hand,

$$
(2 \sqrt{S})^{-1}\|f\|_{1} \geq(2 \sqrt{S})^{-1}\|f\|_{1}\|g\|_{\infty} \geq\left\langle g,(2 \sqrt{S})^{-1} f\right\rangle .
$$

Thus $\|f\|_{1} \geq 4^{-1} c \sqrt{S}$; equivalently

$$
\left(\sum_{j=1}^{\infty}\left\|\hat{f}\left(n_{k}\right)\right\|^{2}\right)^{1 / 2} \leq 4 c^{-1}\|f\|_{1} .
$$

Corollary 3.3. If $1 \leq p \leq 2$ then $S_{p}$ is a Paley space.

Proof. For $1<p \leq 2$ the dual $\left(S_{p}\right)^{*}=S_{p^{\prime}}$ is of type 2 (cf. [T-J]). $S_{1}$ is the dual of the $C^{*}$-algebra of all compact operators on $l^{2}$ so we can apply Theorem 3.3. Alternatively we can use Theorem 2.2 and Proposition 3.3.

\section{REMARKS AND UNSOLVED PROBLEMS}

4.1 Paley spaces versus spaces of $\left(H^{1}-l^{1}\right)$-Fourier type. We do not know examples of Paley spaces which are not of $\left(H^{1}-l^{1}\right)$-Fourier type. We do not even know whether for every Paley space the vector-valued Hardy inequality holds.

The vector-valued Hardy inequality is not a "formal consequence" of the vector-valued Paley inequality in a natural sense described next.

Let $P M \cdot l^{2}$ be the class of scalar sequences $m=\left(m_{j}\right)$ such that $m_{j}=a_{j} s_{j}$ for $j=0,1, \ldots$ for some $\left(a_{j}\right) \in P M$ and $\left(s_{j}\right) \in l^{2}$. We have

$$
\left(m_{j}\right) \in P M \cdot l^{2} \quad \text { iff } \sum_{k=1}^{\infty}\left(\sum_{2^{k-1}}^{2^{k}-1}\left|m_{j}\right|\right)^{2}<\infty,
$$

because

$$
\left(a_{j}\right) \in P M \quad \text { iff } \sup _{k \geq 1} \sum_{2^{k-1}}^{2^{k}-1}\left|a_{j}\right|^{2}<\infty .
$$

Clearly, if $X$ is Paley space then for every $m \in P M \cdot l^{2}, m_{x}$ is $(1,1)$ bounded. On the other hand, the Hardy multiplier $\left((j+1)^{-1}\right)_{j \geq 0}$ does not belong to $P M \cdot l^{2}$, because $\sum_{2^{k-1}}^{2^{k}-1}(j+1)^{-1} \geq \frac{1}{2}$ for $k=1,2, \ldots$.

4.2. More characterizations of Banach spaces with type $>1$. Propositions 3.4 and 3.5 can be modified to obtain yet another characterization of Banach spaces with nontrivial type.

Proposition 4.1. A Banach space $X$ has type $>1$ iff for some $p \geq 2$ there is $C>0$ such that for every 2-lacunary sequence $\left(n_{k}\right)$

$$
\left(\sum_{k \geq 1}\left\|\hat{f}\left(n_{k}\right)\right\|^{p}\right)^{1 / p} \leq C\|f\|_{1, \text { at }} \text { for } f \in H_{X}^{1, \text { at }} .
$$

Moreover, (4.3) holds iff $p^{\prime}$ is the type of $X^{*}$. 
For the necessity of (4.3) first observe that if $X$ has type $>1$, then $X^{*}$ has type $>1$, say $p^{\prime}$ (cf. [M-Pi]). Next we essentially repeat the argument of Proposition 3.4. Conversely, if (4.3) holds for some $p \geq 2$ then a similar argument to that of Proposition 2.5 yields that $X^{*}$ has type $p^{\prime}$. Thus $X$ has type $>1$.

After reading the preliminary version of this paper, G. Pisier has kindly communicated to us that another modification of the proof of Proposition 3.4 yields

Proposition 4.2. A Banach space $X$ has type $>1$ iff there is a constant $C>0$ such that

$$
\left(\int_{0}^{1}\left\|\sum_{k=1}^{\infty} r_{k}(t) \hat{f}\left(2^{k}\right)\right\|^{2} d t\right)^{1 / 2} \leq C\|f\|_{1, \text { at }} \text { for } f \in H_{X}^{1, \text { at }},
$$

where $\left(r_{k}\right)$ is the Rademacher system.

4.3. (1, 2)-bounded multipliers from $H_{L^{p}}^{1}$ into $l_{L^{p}}^{2}$. We do not have a satisfactory description of scalar sequences $m$ such that $m_{L^{p}}$ is $(1,2)$-bounded. To formulate our conjecture we first introduce new classes of sequences.

Let $2 \leq r \leq \infty$. Let $P M_{r}$ be the class of scalar sequences $m=\left(m_{j}\right)_{j}$ such that $\sigma_{r}(m)<\infty$, where

$$
\begin{gathered}
\sigma_{r}(m)=\left(\left|m_{0}\right|^{r}+\sum_{k=1}^{\infty}\left(\sum_{2^{k-1}}^{2^{k}-1}\left|m_{j}\right|^{2}\right)^{r / 2}\right)^{1 / r} \text { for } 2 \leq r<\infty ; \\
\sigma_{\infty}(m)=\max \left(\left|m_{0}\right|, \sup _{k \geq 1}\left(\sum_{2^{k-1}}^{2^{k}-1}\left|m_{j}\right|^{2}\right)^{1 / 2}\right) .
\end{gathered}
$$

By (4.2), $P M_{\infty}=P M$ as classes of sequences, i.e. $\sigma_{\infty}$ and $\sigma$ are equivalent norms. Clearly $P M_{2}$ coincides with $l^{2}$. Thus, if $m \in P M_{2}$ then $m_{x}$ is $(1,2)$ bounded for every Banach space $X$. Furthermore, it follows from Proposition 1.1 that if either $X=c_{0}$ or $X=L^{1} / H_{0}^{1}$ then $m_{x}$ is $(1,2)$-bounded iff $m \in$ $P M_{2}$.

Conjecture. Let $E_{p}$ denote either an infinite $L^{p}(\mu)$-space or $S_{p}, 1 \leq p<\infty$. Let $r \geq 2$ so that $r^{-1}=\max \left(2^{-1}-p^{-1}, 0\right)$. Then

(4.4) $m \in P M_{r}$ iff $m_{E_{p}}$ is $(1,2)$-bounded.

In fact, what is left is the validity of the implication: “ $m_{E_{p}}(1,2)$-bounded $\Rightarrow m \in P M_{1 / 2-1 / p}$ " for $p>2$.

If $1 \leq p \leq 2$ then, by Corollaries 3.2 and $3.3, E_{p}$ is Paley space. Thus, (4.4) is a consequence of $(3.1)$, because if $m_{X}$ is $(1,2)$-bounded for some Banach space $X$ then $m \in P M=P M_{\infty}$. 
We also have

Proposition 4.3. Let $2<p<\infty$. Let $m \in P M_{1 / 2-1 / p}$. Then $m_{E_{p}}$ is $(1,2)$ bounded.

Outline of the proof. It is well known that $\left(E_{p}\right)^{\prime}$ can be identified with $E_{p^{\prime}}$. Furthermore, for $p>2, E_{p^{\prime}}$ is of type $p^{\prime}$ (cf. e.g. [M-Pi and TJ]). Thus the desired conclusion follows from the next two facts.

Corollary 4.1. If $X^{*}$ is of type $p^{\prime}>1$ and if $r$ satisfies $r^{-1}-2^{-1}=p^{-1}$, then there is a positive $C$ such that for every $m \in P M_{r}$ and every $f \in H_{X}^{1}$, at,

$$
\left(\sum_{j>0}\|\hat{f}(j)\|^{2}\left|m_{j}\right|^{2}\right)^{1 / 2} \leq C \sigma_{r}(m)\|f\|_{1, \text { at }} .
$$

Corollary 4.2. If $X^{*}$ is of type $p^{\prime}>1$ and $m \in P M_{r}$ with $r^{-1}=2^{-1}-p^{-1}$ then $m_{x}$ is $(1,2)$-bounded.

Proof of Corollary 4.1. Fix $f \in H_{X}^{1}$, at and pick $n_{k}$ for $k=1,2, \ldots$ so that $2^{k-1} \leq n_{k}<2^{k}$ and $\left\|\hat{f}\left(n_{k}\right)\right\|=\max \left\{\|\hat{f}(j)\|: 2^{k-1} \leq j<2^{k}\right\}$. Then $\left(n_{k}\right)$ is the union of two 2-lacunary sequences. Now using Proposition 4.1 and the Hölder inequality we get (4.5).

Corollary 4.2 follows from Corollary 4.1 and Theorem 1.1.

4.4. Paley spaces versus 2 -uniformly $P L$ convexifiable spaces. Comparing results of $\S 3$ with results in [D-Ga-TJ] (cf. also [B-D]), it is natural to ask what is the relationship between Paley spaces and 2-uniformly PL convexifiable spaces.

Recall that a Banach space $X$ is 2-uniformly PL convexifiable provided it has an equivalent norm $\|\cdot\|$ such that for some $c>0$

$$
(2 \pi)^{-1} \int_{-\pi}^{\pi}\left\|x e^{i t} y\right\|^{2} d t \geq\|x\|^{2}+c^{2}\|y\|^{2} .
$$

After this paper had been submitted for publication, J. Bourgain communicated to us an example of Paley space which is not 2-uniformly convexifiable. On the other hand, G. Pisier observed that every 2-PL convexifiable space is a Paley space. Combining Pisier's result with [D-Ga-TJ, Theorem 4.3] (due to U. Haagerup), one gets an alternative proof of our Theorem 3.3.

4.5. More about preduals of $C^{*}$-algebras. After this paper had been submitted for publication, U. Haagerup and G. Pisier [Ha-Pi] generalized Theorem 2.3 (the noncommutative factorization theorem) to the case of an arbitrary $C^{*}$ algebra. As a consequence they generalized our Theorem 2.2 to the following: Every predual of a $C^{*}$-algebra is of $\left(H^{1}-l^{1}\right)$-Fourier type; in particular for every predual of a $C^{*}$-algebra the vector-valued Hardy inequality holds. The same result was independently obtained by $\mathrm{P}$. Muhly [Mu]. For related results see also $\mathrm{Xu}[\mathrm{X} 1, \mathrm{X} 2]$ and [Pi2].

Finally observe that our Theorem 3.3 combined with Proposition 1.1 implies the result due to Pisier (cf. [D-Ga-TJ, Theorem 6.3 and Corollary 6.4]) that 
the disc algebra is not the continuous image of any $C^{*}$-algebra. The approach via the vector-valued Paley inequality seems to be slightly simpler than that presented in [D-Ga-TJ]. This approach was also proposed by G. Pisier who knew a long time ago that the trace class $S_{1}$ and the dual of $B\left(l^{2}\right)$ are Paley spaces (private discussion in Georgenthal in 1981).

\section{REFERENCES}

[B1] J. Bourgain, Vector-valued singular integrals and the $H^{1}-B M O$ duality, Probability Theory and Harmonic Analysis (J.-A. Chao and W. A. Woyczynski, ds.), Dekker, New York and Basel, 1986, pp. 1-19.

[B2] _ New Banach space properties of the disc algebra and H, Acta Math. 152 (1984), 1-48.

[B3] _ _ A Hausdorff-Young inequality for B-convex Banach spaces, Pacific J. Math. 101 (1982), 255-262.

[B4] _ Vector-valued Hausdorff-Young inequality and applications, Geometric Aspects in Functional Analysis, Israel Seminar (GAFA) 1986-1987, Lecture Notes in Math., vol. 1317, Springer-Verlag, pp. 239-249.

[B-D] J. Bourgain and W. J. Davis, Martingale transforms and complex uniform convexity, Trans. Amer. Math. Soc. 294 (1986), 501-515.

[Be-Gi-Mu] E. Berkson, T. A. Gillespie, and P. S. Muhly, Abstract spectral decompositions guaranteed by the Hilbert transform, Proc. London Math. Soc. (3) 53 (1986), 489-517.

[Bg-Lö] J. Berg and J. Löfström, Interpolation spaces. An introduction, Springer-Verlag, Berlin, Heidelberg, and New York, 1976.

[Bo-Dn] F. F. Bonsall and J. Duncan, Complete normed algebras, Springer-Verlag, Berlin and New York, 1973.

[Du] P. L. Duren, Theory of $H^{p}$ spaces, Academic Press, New York, 1970.

[D-Ga-TJ] W. J. Davis, D. J. H. Garling, and N. Tomczak-Jaegermann, The complex convexity of quasi-normed linear spaces, J. Funct. Anal. 55 (1984), 110-150.

[G-K] I. C. Gohberg and M. G. Krein, Theory and applications of Volterra operators in Hilbert space, Transl. Math. Monographs, no. 24, Amer. Math. Soc., Providence, R.I., 1970.

[GC-Ru] J. Garcia Cuerva and J. L. Rubio de Francia, Weighted norm inequalities and related topics, North-Holland, Amsterdam, 1985.

[H] P. R. Halmos, A Hilbert space problem book, Van Nostrand, Princeton, N.J., 1967.

[Hu-Pi] U. Haagerup and G. Pisier, Factorization of analytic functions with values in non-commutative $L_{1}$-spaces and applications, Canad. J. Math. (to appear).

[Kh] J.-P. Kahane, Some random series of functions, Cambridge Univ. Press, Cambridge, 1985.

[L-R] J. Lindenstrauss and H. P. Rosenthal, The $\mathscr{L}_{p}$ spaces, Israel J. Math. 7 (1969), 325-349.

[L-T] J. Lindenstrauss and L. Tzafriri,Classical Banach spaces. II, Springer-Verlag, New York, 1979.

[M-Pi] B. Maurey and G. Pisier, Séries de variables aléatoires vectorielles indépendantes et propriétés géométriques des espaces de Banach, Studia Math. 58 (1976), 45-90.

[MI] M. Milman, Complex interpolation and geometry of Banach spaces, Ann. Mat. Pura Appl. (4) 136 (1984), 317-328.

[Mu] P. S. Muhly, Fefferman spaces and $C^{*}$-algebras, Contemp Math., vol. 85, Amer. Math. Soc., Providence, R. I., 1989, pp. 371-385.

[P] A. Pelczynski, On commensurate sequences of characters, Proc. Amer. Math. Soc. 104 (1988), 525-531.

[Pa] R. E. A. C. Paley, On Lacunary coefficients of power series, Ann. of Math. 34 (1936), 615616. 
[Pee] J. Peetre, Sur la transformation de Fourier des fonctions à valeurs vectorielles, Rend. Sem. Mat. Univ. Padova 42 (1969), 15-26.

[Pi1] G. Pisier, Les inégalités de Khinchine-Kahane d'après C. Borel, Séminaire sur le Géométrie d'Espaces de Banach 1977-1978, Exposé VII, École Polytechnique, Centre de Mathématiques, 1978.

[Pi2] - Factorization of operator-valued analytic functions, Adv. in Math. (to appear).

[R] H. P. Rosenthal, On subspaces of $L^{p}$, Ann. of Math. (2) 97 (1973), 344-373.

[S] D. Sarason, Generalized interpolation in $H^{\infty}$, Trans. Amer. Math. Soc. 127 (1967), 179203.

[SM] B. Smith, Two trigonometric designs: One-sided Riesz products and Littlewood products, General Inequalities 3 (E. F. Beckenbach and W. Walter, eds.), Birkhäuser-Verlag, Basel, 1983, pp. 141-148.

[Sz-W] S. J. Szarek and T. Wolniewicz, A proof of Fefferman's theorem on multipliers, Inst. of Math., Polish Acad. Sci., Preprint No. 209, Warzawa, 1980.

[TJ] N. Tomczak-Jaegermann, On the moduli of smoothness and the Rademacher averages of the trace classes $S_{p} \quad(1 \leq p<\infty)$, Studia Math. 50 (1974), 163-182.

[X1] Q. Xu, Inégalités pour les martingales de Hardy et renormage des espaces quasi normés, C. R. Acad. Sci. Paris Sér. I Math. 306 (1988), 601-604.

[X2] __ Application du théorème de factorisation pour des fonctions à valeurs opérateurs, Studia Math. (to appear).

Departamento de Matematicas, Universidad de Zaragoza, Facultad de Ciencias, Zaragoza 500009, SPAin

Department of Mathematics, University of Illinols at Urbana-Champaign, 1409 West Green Street, Urbana, ILLINOIS 61801

Institute of Mathematics, Polish Academy of Sciences, Sniadeckich 8, Ip., 00950 Warzawa, Poland 